\title{
A framework for ML estimation of parameters of (mixtures of) common reaction time distributions given optional truncation or censoring
}

\author{
CONOR V. DOLAN, HAN L. J. VAN DER MAAS, and PETER C. M. MOLENAAR \\ University of Amsterdam, Amsterdam, The Netherlands
}

\begin{abstract}
We present a framework for distributional reaction time (RT) analysis, based on maximum likelihood (ML) estimation. Given certain information relating to chosen distribution functions, one can estimate the parameters of these distributions and of finite mixtures of these distributions. In addition, left and/or right censoring or truncation may be imposed. Censoring and truncation are useful methods by which to accommodate outlying observations, which are a pervasive problem in RT research. We consider five RT distributions: the Weibull, the ex-Gaussian, the gamma, the log-normal, and the Wald. We employ quasi-Newton optimization to obtain ML estimates. Multicase distributional analyses can be carried out, which enable one to conduct detailed (across or within subjects) comparisons of RT data by means of loglikelihood difference tests. Parameters may be freely estimated, estimated subject to boundary constraints, constrained to be equal (within or over cases), or fixed. To demonstrate the feasibility of ML estimation and to illustrate some of the possibilities offered by the present approach, we present three small simulation studies. In addition, we present three illustrative analyses of real data.
\end{abstract}

The aim of the present paper is to present a general framework for distributional reaction time (RT) analysis, based on maximum likelihood (ML) estimation. The importance of distributional analyses as a method for characterizing the properties of empirical RT distributions is generally recognized (Heathcote, Popiel, \& Mewhort, 1991; Hockley, 1984; Luce, 1986; Ratcliff, 1979, 1993; Ratcliff \& Murdock, 1976; Ratcliff, Van Zandt, \& McKoon, 1999; Ulrich \& Miller, 1994). A distributional analysis involves three steps: (1) determination of a suitable theoretical distribution function to model or approximate the observed RT distribution (e.g., Logan, 1992; Van Zandt \& Ratcliff, 1995), (2) estimation of the parameters of this distribution, and (3) evaluation of the fit of the theoretical distribution to the data. The determination of a suitable distribution may be approached from two perspectives. On the one hand, one may want to derive a distribution from first principles concerning the psychological process that generates the RTs (Luce, 1986). On the other hand, and more

The research of C.V.D. was made possible by a fellowship from the Royal Dutch Academy of Arts and Sciences. We thank Wulfert van den Brink for free access to his statistics library, Denis Cousineau for his comments and feedback concerning our computer program, Eric-Jan Wagenmakers for his comments on an earlier version of this paper and his general encouragement, and Susanne Hammen and Verena Schmittmann for pointing out several errors. Wery van den Wildenberg kindly contributed data used in the first real-data illustration. Two rounds of detailed reviews by Andrew Heathcote and two anonymous referees resulted in many improvements. Correspondence concerning this paper should be directed to C. V. Dolan, Developmental Psychology, Department of Psychology, University of Amsterdam, Roetersstraat 15, 1018WB Amsterdam, The Netherlands (e-mail: conor@psy.uva.nl). pragmatically, one may simply want a distribution that provides an adequate description of the observed distribution. The ex-Gaussian is often used to this end (Heathcote, 1996; Schnipke \& Scrams, 1997).

The second step, estimation of parameters, is the subject of the present paper. Estimation of the parameters of RT distributions poses several problems. In estimating parameters of a given distribution function, it is important to take account of possible outlying RTs. Within the context of a distributional analysis, these may be accommodated by means of the imposition of censoring (Azzelini, 1996, pp. 26-27; Ulrich \& Miller, 1994) or truncation (Ratcliff, 1993). We will use the term right (left) truncation, or censoring, to refer to the treatment of outlying slow (fast) RTs. Truncation involves discarding RTs beyond chosen thresholds. No assumptions are made concerning the distribution of the discarded observations. The distribution function is renormalized, so that it integrates to unity, and the renormalized distribution is used in ML estimation. Only the retained RTs are used to estimate the parameters. Censoring likewise involves discarding RTs beyond chosen thresholds. However, the number of discarded RTs is supposed to be consistent with the hypothesized distribution of the retained RTs. Ulrich and Miller (1994) have shown that censoring is computationally feasible and that it works well in practice. As compared with censoring, truncation requires much larger samples to work well (Ratcliff, 1993; Ulrich \& Miller, 1994).

In addition to the accommodation of outliers, it may be desirable to model hypothesized heterogeneity, in the psychological process that generates RTs, by specifying a mixture of distributions (Van Zandt \& Ratcliff, 1995; Yantis, Meyer, \& Smith, 1991). Such heterogeneity may arise 
when two or more distinct processes (e.g., two different strategies of responding) generate the RTs. Interestingly, Ulrich and Miller (1994) related outlying RTs to mixture distributions by supposing that the outliers (e.g., extremely fast RTs) were generated by an extraneous process (fast guessing), whereas the other RTs were generated by the process in which they were interested. As Ulrich and Miller (1994) pointed out, an actual mixture modeling approach to accommodate outliers does not appear to be feasible, since outliers are small in number and may be generated by a variety of mechanisms, which give rise to unknown distributions.

The problem of obtaining estimates in distributionalRT analyses is complicated by the imposition of truncation or censoring in single-component or finite mixture distribution. Van Zandt (2000) has provided an in-depth and clear account of methods of estimation in single-componentRT distributions, without censoring or truncation. She considered both nonparametric estimates of the distributions, such as histograms and kernel density estimates, and the parametric estimations of parameters of selected theoretical distributions. Parametric parameter estimation was achieved by ML estimation and by least-squares fits of the theoretical distributions to various empirical estimates of the distributions. Generally, in terms of efficiency and unbiasedness, ML emerged as the best method of estimating parameters.

The main aim of the present paper is to present a framework for ML estimation of RT distribution parameters. We will demonstrate that, with certain information relating to the theoretical distribution functions, one can readily assemble the loglikelihood function for (mixtures of) common RT distributions, given optional truncation or censoring. The required information relates to distribution function values, function derivatives, and integrals. We limit our attention to ML estimation. Van Zandt (2000) and Heathcote (1996) have provided clear explanations of the method of ML estimation. Azzelini (1996) has provided a comprehensive technical treatment. Generally, if certain regularity conditions are met, ML estimates have favorable characteristics, such as efficiency and asymptotic unbiasedness. In addition, standard errors of estimates are readily available, and hypotheses concerning the parameters may be tested by loglikelihood difference (LD) tests (Azzelini, 1996). In the case of mixtures, it is known that ML estimation may run into problems, owing to singularities in the loglikelihood function, and that certain LD tests are invalid (Everitt \& Hand, 1981). Also, as was pointed out by Heathcote, in the case of certain RT distributions, similar problems may occur.

Careful bookkeeping of the required information of each theoretical distribution function is all that is required to arrive at the present comprehensive approach to ML parameter estimation. Of course, this bookkeeping and the maximization of loglikelihood functions can be left to the computer. The present approach has the advantage that it covers a large number of possible situationsnamely, single-component or mixture distributions, which are subject to optional (left and/or right) truncation or cen- soring. In addition, the present approach lends itself readily to multicase analyses, since loglikelihoods may be summed over cases. This, in combination with the facility to maximize loglikelihoods subject to parameter equality constraints on the parameters, enables one to carry out detailed (across or within subjects) comparisons of observed RT distributions, using LD tests (Azzelini, 1996).

In the next section, we will provide the details of the present framework and the various cases that it can handle. In this part of the presentation, we will not actually consider any particular distribution.

\section{REQUIRED INFORMATION}

As was mentioned, we limit our attention to estimation of parameters by maximization of the loglikelihoodfunction. Maximization of a function may be achieved with a variety of methods (Gill, Murray, \& Wright, 1981). Here, we rely exclusively on the quasi-Newton algorithm (Gill et al., 1981) with exact first-order derivatives. We therefore require generic expressions for function values and derivatives.

Consider a mixture distribution consisting of $M$ distinct components. Let $\theta_{i}$ denote the $q$-dimensional vector of unknown parameters of the $i$ th component distribution ( $\mathrm{i}=1 \ldots \mathrm{M}$ ), let $\mathbf{p}$ denote the vector of unknown $M$ mixing proportions, and let $p_{i}$ denote the $i$ th component of $\mathbf{p}$ $\left(\Sigma \mathbf{p}_{i}=1\right)$. Let $x_{j}$ denote the $j$ th observed RT of a given subject. To specify loglikelihood functions and firstorder derivatives, we require four pieces of information, which are summarized in Table 1 (Table 1 contains additional notation, which is introduced to ease presentation). These are denoted as follows: $\mathbf{f}_{i}\left(x_{j} ; \theta_{i}\right)$ (the $i$ th component distribution function evaluated at $\left.x_{j}\right) ; \mathbf{F}_{i}\left(T_{k} ; \theta_{i}\right)$ (the $i$ th component cumulative distribution function evaluated from $-\infty$ to $T_{k}$ ) - that is,

$$
\int_{-\infty}^{T_{k}} \mathbf{f}_{i}\left(x ; \theta_{i}\right) d x=\mathbf{F}_{i}\left(T_{k} ; \theta_{i}\right) ;
$$

$\mathbf{f}_{i}^{\bullet}\left(x_{j} ; \theta_{i}\right)$ (the $q$-dimensional vector of derivatives of the $i$ th distribution function with respect to the parameters $\theta_{i}$, evaluated at $x_{j}$ ) — that is,

$$
\partial \mathbf{f}_{i}\left(x_{j} ; \theta_{i}\right) / \partial \theta_{i}=\mathbf{f}_{i}^{\bullet}\left(x_{j} ; \theta_{i}\right) ;
$$

and $\mathbf{F}_{i}^{\bullet}\left(T_{k} ; \theta_{i}\right)$ [the $q$-dimensional vector of integrals of the derivatives $\mathbf{f}_{i}^{\circ}\left(x_{j} ; \theta_{i}\right)$, evaluated from $-\infty$ to $T_{k}$ ] that is,

$$
\int_{-\infty}^{T_{k}} \mathbf{f}_{i}^{\bullet}\left(x_{j} ; \theta_{i}\right) d x=\partial \mathbf{F}_{i}\left(T_{k} ; \theta_{i}\right) / \partial \theta_{i}=\mathbf{F}_{i}^{\bullet}\left(T_{k} ; \theta_{i}\right) .
$$

Note that we evaluate integrals from minus infinity to a given upper bound $T_{k}$. In modeling RT distributions, the lower bound may be zero or may be dictated by a parameter of the distribution.

In addition, we denote the finite mixture distribution (evaluated at $\left.x_{j}\right) \sum \mathbf{p}_{i} \mathbf{f}_{i}\left(x_{j} ; \theta_{i}\right)$, consisting of $M$ components, as $\mathbf{g}\left(x_{j} ; \theta, \mathbf{p}\right)$, and its integral as $\mathbf{G}\left(T_{k} ; \theta, \mathbf{p}\right)$ - that is, $\sum \mathbf{p}_{i} \mathbf{F}_{i}\left(T_{k} ; \theta_{i}\right)$ [evaluated from $-\infty$ to threshold $T_{k}$; the 
Table 1

Required Information

\begin{tabular}{|c|c|}
\hline \multicolumn{2}{|r|}{ initions: observed } \\
\hline$x_{j}$ & $\begin{array}{l}\text { served } j \text { th } \mathrm{RT} \text { of a given subject in a given } \\
\text { in }\end{array}$ \\
\hline$\theta_{i}$ & $\begin{array}{l}\text { ctor of unknown parameters of distribution } i(i= \\
\text {..M) }\end{array}$ \\
\hline$\theta$ & vector of unknown parameters $\theta=\left[\theta_{1}^{t}, \theta_{2}^{t}, \ldots, \theta_{\mathrm{M}}^{t}\right]^{t}$ \\
\hline $\mathbf{p}_{i}$ & ponent of $\mathbf{p}=\left[p_{1}, p_{2}, \ldots, p_{\mathrm{M}}\right]^{t}$ \\
\hline $\mathbf{p}$ & 1 vector of mixing pro \\
\hline \multicolumn{2}{|c|}{ Required information } \\
\hline & $\begin{array}{l}\text { distribution function } i \text { of } \mathrm{RT} x_{j} \text { (scalar), comprising } \\
q \text { parameters (in a mixture, this is a component dis- } \\
\text { tribution) }\end{array}$ \\
\hline $\mathbf{F}_{i}\left(T_{k} ; \theta_{i}\right)$ & $\begin{array}{l}\text { of } \mathbf{f}_{i}\left(x_{j} ; \theta_{i}\right) \text {, evaluated from }-\infty \text { to threshold } \\
\text { ir })^{\dagger}\end{array}$ \\
\hline $\mathbf{f}_{i}^{*}\left(x_{j} ; \theta_{i}\right)$ & $\begin{array}{l}\text { derivative of function } \mathbf{f}_{i}\left(x_{j} ; \theta_{i}\right) \text { with respect to param- } \\
\text { eter } \theta_{i}\left(q \text {-dimensional vector } \text { - that is, } \partial \mathbf{f}_{\mathbf{f}}\left(x_{i} ; \theta_{j}\right) / \partial \theta_{\text {- }}\right.\end{array}$ \\
\hline $\mathbf{F}_{i}^{\bullet}\left(T_{k} ; \theta_{i}\right)$ & $\begin{array}{l}\text { integral of } \partial \mathbf{f}_{i}\left(x_{j} ; \theta_{i}\right) / \partial \theta_{i} \text { evaluated from }-\infty \text { to } \\
\text { threshold } T_{k} \text { [this is equivalent to } \partial \mathbf{F}_{i}\left(T_{k} ; \theta_{i}\right) / \partial \theta_{i} \\
q \text {-dimensional vector] } \dagger\end{array}$ \\
\hline \multicolumn{2}{|l|}{ Derived entities } \\
\hline & \\
\hline $\mathbf{G}\left(T_{k} ; \theta, \mathbf{p}\right)=$ & $\begin{array}{l}\Sigma p_{i} \mathbf{F}_{i}\left(T_{k} ; \theta_{i}\right), \text { integral of } \mathbf{g}\left(x_{j} ; \theta, \mathbf{p}\right), \text { evaluated from } \\
-\infty \text { to threshold } T_{k}^{\dagger}\end{array}$ \\
\hline
\end{tabular}

*Throughout,we do not estimate $p_{\mathrm{M}}$ as a free parameter; rather, it is derived as follows: $p_{\mathrm{M}}=1-p_{1}-p_{2}-\ldots-p_{\mathrm{M}-1}$. We take infinity to be the lower bound. In modeling RT distributions, this bound may equal zero or may equal a parameter of the distribution. For instance, a natural lower bound is the shift parameter, $\xi$, in the Wald (see Equation 5), log-normal (Equation 4), gamma (Equation 3), and Weibull (Equation 2) distributions.

vector $\theta$ equals $\left.\left(\theta_{1}^{t}, \theta_{2}^{t} \ldots \theta_{M}^{t}\right)^{t}\right]$. These are derived entities, which depend on the information defined in Table 1. Given this information, we can assemble the loglikelihood function and required derivatives for a variety of cases involving mixtures and optional censoring or truncation.

Tables $2 \mathrm{~A}$ to $2 \mathrm{E}$ contain the distribution functions, $\log$ likelihood functions, and derivatives for single-component and finite mixture distributions, given optional left and/or right censoring or truncation. As will be discussed in more detail below, the definitions of a censored distribution and a truncated distribution differ importantly. Consider, for instance, truncation and censoring to the right at point $T_{\mathrm{R}}$ in a single-component distribution. Given right truncation (Table 2B), the distributionfunction is $\mathbf{f}_{i}\left(x_{j} ; \theta_{i}\right) /\left[\mathbf{F}_{i}\left(T_{\mathrm{R}} ; \theta_{i}\right)\right]$, where $x_{j}<T_{\mathrm{R}}$. Here, the denominator, $\left[\mathbf{F}_{i}\left(T_{\mathrm{R}} ; \theta_{i}\right)\right]$, renormalizes the function $\mathbf{f}_{i}\left(x_{j} ; \theta_{i}\right)$ so that the integral $\int\left\{\mathbf{f}_{i}\left(x_{j} ; \theta_{i}\right) /\left[\mathbf{F}_{i}\left(T_{\mathrm{R}} ; \theta_{i}\right)\right]\right\}$, evaluated from $-\infty$ (or a natural lower bound) to $T_{\mathrm{R}}$, equals one, as required. In the case of censoring, the distribution function is defined as $\mathbf{f}_{i}\left(x_{j} ; \theta_{i}\right)$ for $x_{j}<T_{\mathrm{R}}$. If $x_{j} \geq T_{\mathrm{R}}, x_{j}$ is assigned the value $T_{\mathrm{R}}$ (Greene, 1993,pp. 691-692). The probability of $x_{j} \geq T_{\mathrm{R}}$ then equals $1-\left[\mathbf{F}_{i}\left(T_{\mathrm{R}} ; \theta_{i}\right)\right]$, and so the contribution to the $\operatorname{loglikelihood}$ of the $N_{\mathrm{R}}$ censored observations equals $N_{\mathrm{R}}$ $\log \left[1-\mathbf{F}_{i}\left(T_{\mathrm{R}} ; \theta_{i}\right)\right]$ (Table 2C). Censoring and truncation, as applied in the context of distributional RT analysis, will be discussed in more detail below.

So far, we have not considered any particular distribution. Indeed, the generality of the present approach lies partly in the fact that $\mathbf{f}_{i}\left(x_{j} ; \theta_{i}\right)$ may represent any distribu- tion function. In developing a FORTRAN program on the basis of the present approach, we considered six RT distributions. These distributions will be briefly discussed below.

\section{COMMON RT DISTRIBUTIONS}

Various distribution functions have been suggested for RT data. Here, we consider the following six: the normal, the Weibull, the gamma, the log-normal, the Wald, and the ex-Gaussian distributions. Since these have been discussed both in the RT literature (Luce, 1986; Ulrich \& Miller, 1994; van Zandt, 2000) and in the statistical literature (Johnson, Kotz, \& Balakrishnan, 1994), we will discuss them briefly. The normal distribution function (Johnson et al., 1994) is defined as follows (we drop the subscript on $x$ ):

$$
f_{N}(x ; \mu, \sigma)=\exp \left[-(x-\mu)^{2} /\left(2 \sigma^{2}\right)\right] /[\sigma \sqrt{(2 \pi)}],
$$

where $\mu$ is the mean and $\sigma$ is the standard deviation. One well-known derivation is that of the sum of a large number of identically and independently distributed random variables. Although Luce (1986) did discuss this distribution, it is considered to be unsuitable for RTs, in view of

Table 2A

Single-Component Distribution $(M=1)$ and Mixture Distribution $(M>1)$
$M=1:$ no truncation/censoring (summation is over all RTs) Distribution function (df): $\quad \mathbf{f}_{i}\left(x_{j} ; \theta_{i}\right)$
Loglikelihood function (LF): $L\left(\theta_{i}\right)=\sum \log \left[\mathbf{f}_{i}\left(x_{j} ; \theta_{i}\right)\right]$
Derivatives (dLF): $\quad \partial L\left(\theta_{i}\right) / \partial \theta_{i}=\sum\left\{\mathbf{f}_{i}^{\circ}\left(x_{j} ; \theta_{i}\right) / \mathbf{f}_{i}\left(x_{j} ; \theta_{i}\right)\right\}$
$M>1$ : no truncation/no censoring (summation is over all RTs)
df: $\quad \mathbf{g}\left(x_{j} ; \theta, \mathbf{p}\right)=\sum p_{i} \mathbf{f}_{i}\left(x_{j} ; \theta_{i}\right)$
LF: $\quad L(\theta, \mathbf{p})=\sum \log \left[\mathbf{g}\left(x_{j} ; \theta, \mathbf{p}\right)\right]$
$\mathrm{dLF}: \partial L(\theta, \mathbf{p}) / \partial \theta_{i}=\sum\left\{p_{i} \mathbf{f}_{i}^{\bullet}\left(x_{j} ; \theta_{i}\right) / \mathbf{g}\left(x_{j} ; \theta, \mathbf{p}\right)\right\}$
$\mathrm{dLF}: \partial L(\theta, \mathbf{p}) / \partial p_{i}=\sum\left\{\left[\mathbf{f}_{i}\left(x_{j} ; \theta_{i}\right)-\mathbf{f}_{M}\left(x_{j} ; \theta_{M}\right)\right] / \mathbf{g}\left(x_{j} ; \theta, \mathbf{p}\right)\right\}^{*}$

*To understand $\partial L(\theta, \mathbf{p}) / \partial p_{i}$ (here and in the tables below), one should bear in mind that in fitting a mixture, we do not estimate $p_{M}$ as a free parameter; rather, it is derived as $p_{M}=1-p_{1}-p_{2}-\ldots-p_{M-1}$.

Table 2B

Single-Component Distribution $(M=1)$, Truncation

Truncation on the left at $T_{\mathrm{L}} ; T_{\mathrm{L}}$ is left, or lower, threshold (summation is over RTs $\left.x_{j}>T_{\mathrm{L}}\right)$

$$
\begin{array}{ll}
\text { df: } & \mathbf{f}_{i}\left(x_{j} ; \theta_{i}\right) /\left[1-\mathbf{F}_{i}\left(T_{\mathrm{L}} ; \theta_{i}\right)\right], x_{j}>T_{\mathrm{L}} \\
\text { LF: } & L\left(\theta_{i}\right)=\sum\left\{\log \left[\mathbf{f}_{i}\left(\mathrm{x}_{j} ; \theta\right)\right]-\log \left[1-\mathbf{F}_{i}\left(T_{\mathrm{L}} ; \theta_{i}\right)\right]\right\} \\
\text { dLF: } & \partial L\left(\theta_{i}\right) / \partial \theta_{i}=\sum\left\{\left[\mathbf{f}_{i}^{\bullet}\left(\mathrm{x}_{j} ; \theta_{i}\right) / \mathbf{f}_{i}\left(\mathrm{x}_{j} ; \theta_{i}\right)\right]-\left(-\mathbf{F}_{i}^{\bullet}\left(T_{\mathrm{L}} ; \theta_{i}\right) /\right.\right. \\
& \left.\left.\left[1-\mathbf{F}_{i}\left(T_{\mathrm{L}} ; \theta_{i}\right)\right]\right)\right\}
\end{array}
$$

Truncation on the right at $T_{\mathrm{R}} ; T_{\mathrm{R}}$ is right, or upper, threshold (summation is over RTs $\left.x_{j}<T_{\mathrm{R}}\right)$

$$
\begin{array}{ll}
\text { df: } & \mathbf{f}_{i}\left(x_{j} ; \theta_{i}\right) /\left[\mathbf{F}_{i}\left(T_{\mathrm{R}} ; \theta_{i}\right)\right], x_{j}<T_{\mathrm{R}} \\
\mathrm{LF}: & L\left(\theta_{i}\right)=\sum\left\{\log \left[\mathbf{f}_{i}\left(x_{j} ; \theta\right)\right]-\log \left[\mathbf{F}_{i}\left(T_{\mathrm{R}} ; \theta_{i}\right)\right]\right\}
\end{array}
$$$$
\mathrm{dLF}: \partial L(\theta) / \partial \theta_{i}=\sum\left\{\mathbf { f } _ { i } ^ { \bullet } \left(\left(x_{j} ; \theta_{i}\right) / \mathbf{f}_{i}\left(x_{j} ; \theta_{i}\right)-\left[\left(\mathbf{F}_{i}^{\bullet}\left(T_{\mathrm{R}} ; \theta_{i}\right) / \mathbf{F}_{i}\left(T_{\mathrm{R}} ; \theta_{i}\right)\right]\right\}\right.\right.
$$

Double truncation at $T_{\mathrm{L}}$ and $T_{\mathrm{R}}$ (summation is over RTs $T_{\mathrm{L}}<x_{j}<T_{\mathrm{R}}$ )

$$
\text { df: } \quad \mathbf{f}_{i}\left(x_{j} ; \theta_{i}\right) /\left[\mathbf{F}_{i}\left(T_{\mathrm{R}} ; \theta_{i}\right)-\mathbf{F}_{i}\left(T_{\mathrm{L}} ; \theta_{i}\right)\right], T_{\mathrm{L}}<x_{j}<T_{\mathrm{R}}
$$$$
\text { LF: } \quad L\left(\theta_{i}\right)=\sum\left\{\log \left[\mathbf{f}_{i}\left(x_{j} ; \theta_{i}\right)\right]-\log \left[\mathbf{F}_{i}\left(T_{\mathrm{R}} ; \theta_{i}\right)-\mathbf{F}_{i}\left(T_{\mathrm{L}} ; \theta_{i}\right)\right]\right\}
$$$$
\mathrm{dLF}: \partial L\left(\theta_{i}\right) / \partial \theta_{i}=\sum\left\{\mathbf { f } _ { i } ^ { \circ } \left(\left(x_{j} ; \theta_{i}\right) / \mathbf{f}_{i}\left(x_{j} ; \theta_{i}\right)-\left[\mathbf{F}_{i}^{\circ}\left(T_{\mathrm{R}} ; \theta_{i}\right)\right.\right.\right.
$$

$$
\left.\left.-\mathbf{F}_{i}^{\bullet}\left(T_{\mathrm{L}} ; \theta_{i}\right)\right] /\left[\mathbf{F}_{i}\left(T_{\mathrm{R}} ; \theta_{i}\right)-\mathbf{F}_{i}\left(T_{\mathrm{L}} ; \theta_{i}\right)\right]\right\}
$$

Note-df, distribution function; LF, loglikelihood function; dLF, derivatives. 
Table 2C

Single-Component Distribution $(M=1)$, Censoring

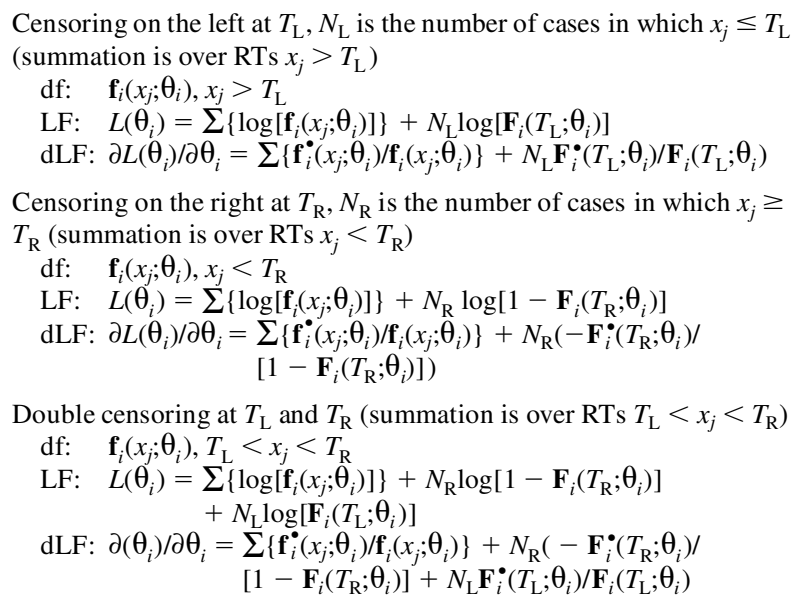

Note-df, distributuion function; LF, loglikelihood function; dLF, derivatives.

Table 2D

Finite Mixture Distribution $(M>1)$, Truncation

$$
\begin{aligned}
& \text { df: } \quad \mathbf{g}\left(x_{i} ; \theta, \mathbf{p}\right) /\left[1-\mathbf{G}\left(T_{\mathrm{L}} ; \theta, \mathbf{p}\right)\right], x_{j}>T_{\mathrm{L}} \\
& \text { LF: } \quad L(\theta, \mathbf{p})=\sum\left\{\log \left[\mathbf{g}\left(x_{j} ; \theta, \mathbf{p}\right)\right]-\log \left[1-\mathbf{G}\left(T_{\mathrm{L}} ; \theta, \mathbf{p}\right)\right]\right\} \\
& \text { dLF: } \partial L(\theta, \mathbf{p}) / \partial \theta_{i}=\Sigma\left\{p_{i} \mathbf{f}_{i}^{*}\left(x_{j} ; \theta_{i}\right) / \mathbf{g}\left(x_{j} ; \theta, \mathbf{p}\right)-\left[-p_{i} \mathbf{F}_{i}^{*}\left(T_{\mathrm{L}} ; \theta_{i}\right)\right] /\right. \\
& \left.\left[1-\mathbf{G}\left(T_{\mathrm{L}} ; \theta, \mathbf{p}\right)\right]\right\} \\
& \text { dLF: } \partial L(\theta, \mathbf{p}) / \partial \mathrm{p}_{i}=\sum\left\{\left[\mathbf{f}_{i}\left(x_{j} ; \theta_{i}\right)-\mathbf{f}_{M}\left(x_{j} ; \theta_{M}\right)\right] / \mathbf{g}\left(x_{j} ; \theta, \mathbf{p}\right)\right. \\
& \left.-\left[\mathbf{F}_{i}\left(T_{\mathrm{L}} ; \theta_{i}\right)-\mathbf{F}_{M}\left(T_{\mathrm{L}} ; \theta_{M}\right)\right] /\left[1-\mathbf{G}\left(T_{\mathrm{L}} ; \theta, \mathbf{p}\right)\right]\right\}
\end{aligned}
$$

Truncation on the right at $T_{\mathrm{R}}\left(T_{\mathrm{R}}\right.$ is right threshold (summation is over RTs $x_{j}<T_{\mathrm{R}}$ )

df: $\quad \mathbf{g}\left(x_{j} ; \theta, \mathbf{p}\right) / \mathbf{G}\left(T_{\mathrm{R}} ; \theta, \mathbf{p}\right), x_{j}<T_{\mathrm{R}}$

LF: $\quad L(\theta, \mathbf{p})=\sum\left\{\log \left[\mathbf{g}\left(x_{j} ; \theta, \mathbf{p}\right)\right]-\log \left[\mathbf{G}\left(T_{\mathrm{R}} ; \theta, \mathbf{p}\right)\right]\right\}$

dLF: $\partial L(\theta, \mathbf{p}) / \partial \theta_{i}=\sum\left\{p_{i} \mathbf{f}_{i}^{*}\left(x_{i} ; \theta_{i}\right) / \mathbf{g}\left(x_{j} ; \theta, \mathbf{p}\right)-p_{i}\right.$ $\left.\mathbf{f}_{i}^{*}\left(T_{\mathrm{R}} ; \theta_{i}\right) / \mathbf{G}\left(T_{\mathrm{R}} ; \theta, \mathbf{p}\right)\right\}$

$\mathrm{dLF}: \partial L(\theta, \mathbf{p}) / \partial p_{i}=\sum\left\{\left[\mathbf{f}_{i}\left(x_{j} ; \theta_{j}\right)-\mathbf{f}_{M}\left(x_{j} ; \theta_{M}\right)\right] / \mathbf{g}\left(x_{j} ; \theta, \mathbf{p}\right)\right.$

$$
\left.-\left[\mathbf{F}_{i}\left(T_{\mathrm{R}} ; \theta_{i}\right)-\mathbf{F}_{M}\left(T_{\mathrm{R}} ; \theta_{M}\right)\right] / \mathbf{G}\left(T_{\mathrm{R}} ; \theta, \mathbf{p}\right)\right\}
$$

Double truncation at $T_{\mathrm{L}}$ and $T_{\mathrm{R}}$ (summation is over RTs $T_{\mathrm{L}}<x_{j}<T_{\mathrm{R}}$ )

df: $\quad \mathbf{g}\left(x_{j} ; \theta, \mathbf{p}\right) /\left[\mathbf{G}\left(T_{\mathrm{R}} ; \theta, \mathbf{p}\right)-\mathbf{G}\left(T_{\mathrm{L}} ; \theta, \mathbf{p}\right)\right], T_{\mathrm{L}}<x_{j}<T_{\mathrm{R}}$

LF: $\quad L(\theta, \mathbf{p})=\Sigma\left\{\log \left[\mathbf{g}\left(x_{j} ; \theta, \mathbf{p}\right)\right]-\log \left[\mathbf{G}\left(T_{\mathrm{R}} ; \theta, \mathbf{p}\right)-\mathbf{G}\left(T_{\mathrm{L}} ; \theta, \mathbf{p}\right)\right]\right\}$

$\mathrm{dLF}: \partial L(\theta, \mathbf{p}) / \partial \theta_{i}=\sum\left\{p_{i} \mathbf{f}_{i}^{*}\left(x_{j} ; \theta_{i}\right) / \mathbf{g}\left(x_{j} ; \theta, \mathbf{p}\right)-p_{i}\left[\mathbf{f}_{i}^{*}\left(T_{\mathrm{R}} ; \theta_{i}\right)\right.\right.$ $\left.\left.-\mathbf{f}_{i}^{\circ}\left(T_{\mathrm{L}} ; \theta_{i}\right)\right] /\left[\mathbf{G}\left(T_{\mathrm{R}} ; \theta, \mathbf{p}\right)-\mathbf{G}\left(T_{\mathrm{L}} ; \theta, \mathbf{p}\right)\right]\right\}$

dLF: $\partial L(\theta, \mathbf{p}) / \partial p_{i}=\sum\left\{\left[\mathbf{f}_{i}\left(x_{j} ; \theta_{i}\right)-\mathbf{f}_{M}\left(x_{j} ; \theta_{M}\right)\right] / \mathbf{g}\left(x_{j} ; \theta, \mathbf{p}\right)\right.$ - $\left[\left\{\mathbf{F}_{i}\left(T_{\mathrm{R}} ; \theta_{i}\right)-\mathbf{F}_{M}\left(T_{\mathrm{R}} ; \theta_{M}\right)\right\}-\left\{\mathbf{F}_{i}\left(T_{\mathrm{L}} ; \theta_{i}\right)\right.\right.$ $\left.\left.\left.-\mathbf{F}_{M}\left(T_{\mathrm{L}} ; \theta_{M}\right)\right\}\right] /\left[\mathbf{G}\left(T_{\mathrm{R}} ; \theta, \mathbf{p}\right)-\mathbf{G}\left(T_{\mathrm{L}} ; \theta, \mathbf{p}\right)\right]\right\}$

Note-df, distribution function; LF, loglikelihood function; dLF, derivatives.

its symmetry. RT data are generally positively skewed and are invariably bounded from below.

The three-parameter Weibull distribution function (Johnson et al., 1994) is defined as follows:

$$
\begin{aligned}
& f_{\mathrm{W}}(x ; \alpha, \gamma, \xi) \\
& \quad=(\gamma / \alpha)[(x-\xi) / \alpha]^{\gamma-1} \exp \left(-[(x-\xi) / \alpha]^{\gamma}\right),
\end{aligned}
$$

where $\alpha$ is the scale parameter $(\alpha>0), \gamma$ is the shape parameter $(\gamma>0)$, and $\xi$ is the shift parameter $(\xi<x)$. The shift parameter determines the position of the distribution on the real line, without changing its form. This distribution is the limit distribution of the minimum of independent, identically distributed variables that are bounded from below. Logan (1992) suggested that the Weibull distribution of RTs follows from the assumptions of his instance theory of automaticity. Colonius (1995) provided the precise conditionsin which the Weibull will obtain. The singleparameter exponential is a special case of this distribution $(\gamma=1, \xi=0)$.

The three-parameter gamma distributionfunction (Johnson et al., 1994) is defined as follows:

$$
\begin{array}{r}
f_{\mathrm{G}}(x ; \alpha, \beta, \xi)= \\
{\left[(x-\xi)^{\beta-1} \exp (-(x-\xi) / \alpha)\right] /[\alpha \beta \Gamma(\beta)],}
\end{array}
$$

where $\Gamma(\beta)$ is the gamma function, $\alpha$ is the scale parameter $(\alpha>0), \beta$ is the shape parameter $(\beta>0)$, and $\xi$ is the shift parameter $(\xi<x)$. The relevance of this distribution lies in the fact that, if $\beta$ assumes integer values, this is the distribution of the sum of $\beta$ independent and identically distributed exponential random variables. As such, it is relevant in models of information processing with $\beta$ successive stages. If the parameter $\beta$ is limited to positive integer values, the distribution is called the special Erlangian distribution (see Ratcliff \& Murdock, 1976; Ulrich \& Miller, 1993; Van Zandt, 2000). The single parameter exponential distribution is a special case of this distribution $(\beta=1, \xi=0)$.

Table 2E

Finite Mixture Distribution $(M>1)$, Censoring

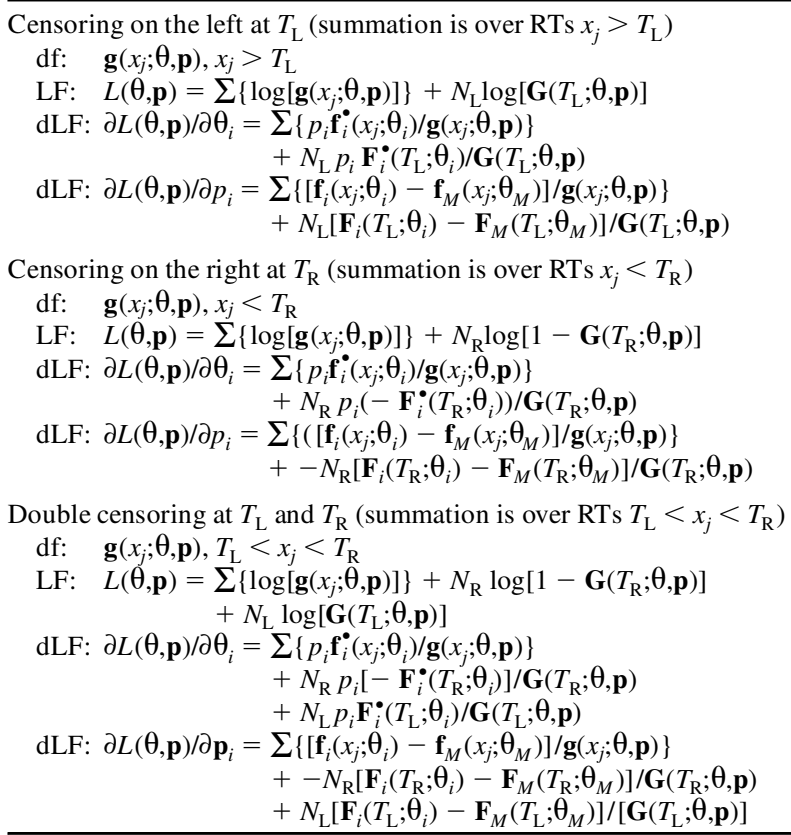

Note—df, distributuion function; LF, loglikelihood function; dLF, derivatives. 
The three-parameter log-normal distribution function (Johnson et al., 1994) is defined as follows:

$$
\begin{aligned}
f_{\mathrm{LN}}(x ; \mu, \sigma, \xi)= & \exp \left(-\left[(\log (x-\xi)-\mu)^{2} /\left(2 \sigma^{2}\right)\right]\right) / \\
& {[(x-\xi) \sqrt{(2 \pi)} \sigma], }
\end{aligned}
$$

where the shift parameter $\xi<x$, and $\sigma>0$. The distribution of the transformed variable $\log (x-\xi)$ is normal, with mean $\mu$ and standard deviation $\sigma$. Luce (1986) presents the log-normal as an ad hoc distribution-that is, without a theoretical derivation. However, Ulrich and Miller (1993) discuss plausible latency mechanisms. See Schnipke and Scrams (1997) for an application.

The three-parameter Wald or inverse normal (Johnson et al., 1994) is defined as follows:

$$
\begin{aligned}
f_{\mathrm{IN}}(x ; \kappa, \lambda, \xi) & \left.=\sqrt{\left[\lambda /(2 \pi)(\mathrm{x}-\xi)^{3}\right)}\right] \\
& \exp \left(-\left[\lambda /\left(2 \kappa^{2}(x-\xi)\right)\right](x-\kappa-\xi)^{2}\right) .
\end{aligned}
$$

This is the distribution of the passage time in a random walk, subject to positive drift, to hit a single numerical (absorbing) barrier (Luce, 1986). The random walk with positive drift represents the accumulation of information, and the actual response occurs when the barrier is hit. Burbeck and Luce (1982) employed this distribution to model simple RTs. Van Zandt and Ratcliff (1995) contrasted this model with the gamma distribution, but they noted that the distribution of RTs generated by these models may be hard to distinguish empirically. Ratcliff et al. (1999) and Van Zandt, Colonius, and Proctor (2000) employed the closely related diffusion model to model two-choice RTs. The diffusion model is characterized by two absorbing barriers. By setting the lower barrier to $-\infty$, the distribution associated with the diffusion model reduces to the Wald (Burbeck $\&$ Luce, 1982). The parameters $\kappa$ and $\lambda$ are related to the physical process in the following manner. Let $\phi$ denote the value of the absorbing boundary, let $\delta$ represent the value of the positive drift, and let $\sigma^{2}$ denote the variance of the normally distributed steps in the random walk. These give rise to parameters of the present formulation: $\kappa=\phi / \delta$ and $\lambda=\phi^{2} / \sigma^{2}$. The parameter $\xi$ shifts the distribution along the real line, without changing its form.

The ex-Gaussian (Heathcote et al., 1991; Hockley, 1984; Hohle, 1965; Ratcliff \& Murdock, 1976) is defined as

$$
\begin{aligned}
f_{\mathrm{EXG}}(x ; \mu, \sigma, \tau)= & (1 / \tau) \exp \left([\mu / \tau]+\left[\sigma^{2} / 2 \tau^{2}\right]\right. \\
& -[x / \tau]) \Phi\left[\left(x-\mu-\left[\sigma^{2} / \tau\right]\right) / \sigma\right],
\end{aligned}
$$

where $\Phi[z]$ is the cumulative standard normal distribution, evaluated from $-\infty$ to $z$. As was explained clearly by Heathcote (1996), the ex-Gaussian is a convolution of the normal (parameters $\mu$ and $\sigma$ ) and the single-parameter exponential (parameter $\tau>0$ ) distribution functions. In the original derivation, the exponential represents decision time, and the normal represents the residual latency, comprising all other processes. Although doubts have been expressed about the validity of this model for RTs, the exGaussian has often been used, since it was known to fit RTs relatively well (Luce, 1986, p. 100; Ratcliff \& Mur- dock, 1976). A limitation of the ex-Gaussian is that the variable $x$ is not restricted to be positive.

Luce (1986, Appendix B) provided an overview of these distributions, including first and second moments and hazard functions. Ulrich and Miller (1994) provided an extensive discussion of the special Erlangian (gamma), the log-normal, and the ex-Gaussian distributions. Heathcote (1996) discussed the ex-Gaussian. Van Zandt (2000) discussed, inter alia, the gamma, ex-Gaussian, Weibull, and Wald distributions.

\section{SOME COMPUTATIONAL/PROGRAMMING ASPECTS}

In developing our own program on the basis of the present framework, we obtained some of the required information (Table 1) from the literature. Notably, Johnson et al. (1994, see also Evans, Hastings, \& Peacock, 2000; Van Zandt, 2000) contains expressions for the cumulative distribution functions. Ulrich and Miller (1994) and Van Zandt (2000) have provided an expression for the cumulative ex-Gaussian distribution. In addition, we used the program MAPLE (e.g., Heck, 1997) to obtain the firstorder derivatives of the distribution functions and cumulative distribution functions with respect to the parameters. MAPLE provides results in the form of FORTRAN code. To illustrate this, we will present the first-order derivative of the Weibull with respect to the parameter $\alpha$, as obtained using MAPLE, in Appendix A.

As was mentioned above, we maximize the loglikelihood function by means of a quasi-Newton algorithm. This is not the only way to maximize a function. Van Zandt (2000), having compared various methods, reported good results with the simplex algorithm, a direct search algorithm that does not require any derivatives. Heathcote (1996) employed both quasi-Newton and simplex algorithms. We use the NPSOL FORTRAN routine (Gill, Murray, Saunders, \& Wright, 1986) to carry out maximization. We provide NPSOL with loglikelihood function values and first-order derivatives of the loglikelihood function with respect to the estimated parameters. Although NPSOL will approximate derivatives if they are not provided, we consistently provide exact derivatives. Exact derivatives increase the speed and the robustness of the optimization process.

The quasi-Newton algorithm requires starting values. In the case of single-component distributions, in contrast to mixtures, starting values may often be obtained by means of moment estimators (Miller \& Miller, 1999). Johnson et al. (1994) have presented these for the distribution functions presented above. Heathcote (1996) and Ulrich and Miller (1994) have presented a moment estimator for the ex-Gaussian. The option to calculate moment estimators in simple cases is included in our program.

Following successful optimization, we employ central finite differences to calculate the matrix of second-order partial derivatives - that is, the observed information matrix (Dennis \& Schnabel, 1983; Dolan \& Molenaar, 1991; Gill et al., 1981). Analytic expressions for this matrix may 
be obtained with MAPLE. Standard errors of ML estimates are the square roots of the diagonals of the inverse of the information matrix (Azzelini, 1996). These standard errors are based on a quadratic approximation to the loglikelihood function at its maximum. A second method by which to obtain an indication of the precision of an ML estimate is to construct loglikelihood profiles (Azzelini, 1996; Venzon \& Moolgavkar, 1988). These may be obtained by fixing the parameter of interest at values increasingly removed from the ML estimate and refitting the model (all other parameters are reestimated). The plot of the fixed values by the changes in loglikelihood provides the profile. The method, which is laborious to carry out by hand, may be programmed (see Neale \& Miller, 1997; Visser, Raijmakers, \& Molenaar, 2000).

The standardized inverse information matrix provides information concerning the correlations among the parameters. The standard errors and parameter correlations provide some insight into the quality of the model fit. For instance, very large standard errors of estimates should not be viewed as acceptable, even though the optimization process has terminated successfully and the parameter estimates appear to be acceptable.

To determine the success of the optimization process, we check that minus the finite-differences information matrix is positive definite and that all gradients are smaller than a predetermined value (e.g., 10E-5). NPSOL provides exit diagnostics based on similar criteria. In calculating the function values and derivatives, we make use of the framework provided in Tables 1-2E. A schematic representation of our routine for calculating the loglikelihood and derivatives will be given in Appendix B.

\section{COMMENTS}

Below, we will present several illustrative analyses. In the present section, we will first provide a number of comments relating to the possibilities and limitations of the present approach.

\section{Outlying Observations in Mixture Distributions}

Often in mixture modeling, components are homogeneous (e.g., mixtures of normals or mixtures of exponentials; see Everitt \& Hand, 1981). We note that the mixture distribution $\mathbf{g}\left(x_{j} ; \theta, \mathbf{p}\right)$ in the present approach need not consist of component distributions $\left[\mathbf{f}_{i}\left(x_{j} ; \theta_{i}\right)\right]$ of the same type. For instance, one may consider a mixture of a Weibull and an exponential distribution (Johnson et al., 1994, p. 690) or a mixture of a Weibull and a Wald distribution (Johnson et al., 1994, p. 287). In the specification of mixtures, care has to be taken that each observation is compatible with each component distribution. This is clear in the definition of the mixture as $\sum \mathbf{p}_{i} \mathbf{f}_{i}\left(x_{j} ; \theta_{i}\right)$, where each component distribution function value $\mathbf{f}_{i}\left(x_{j} ; \theta_{i}\right)$ has to be calculated given the observation $x_{j}$. For instance, in the present approach, numerical problems will occur in estimating parameters of a mixture of, say, a zero mean normal and an exponential distribution. A negative observation cannot belong to the exponential distribution. Similar problems may occur in fitting mixtures of three-parameter Weibull, log-normal, Wald, or gamma distributions, where the shift parameter varies over the component distributions. If the shift parameter is constrained to be equal over the component distributions, this problem does not emerge.

\section{Assumption in Using Censoring to Accommodate Outliers}

As was mentioned above, the imposition of truncation or censoring involves a different treatment of observations beyond the specified threshold(s) (Greene, 1993; Kendall \& Stuart, 1968, p. 541; Ulrich \& Miller, 1994). In the case of censoring, but not of truncation, the excluded observations should be consistent in number with the specified distribution-that is, $\mathbf{f}_{i}\left(x_{j} ; \theta_{i}\right)$ or $\mathbf{g}\left(x_{j} ; \theta, \mathbf{p}\right)$. Note that these numbers $\left(N_{\mathrm{L}}\right.$ and/or $\left.N_{\mathrm{R}}\right)$ feature explicitly in the loglikelihood functions (Tables $2 \mathrm{C}$ and $2 \mathrm{E}$ ). For instance, suppose that one observes 1,000 deviates. One is sure that the values between -2.17 and 2.17 follow the standard normal distribution, and one suspects that observations beyond these thresholds are due to extraneous processes. ML estimation, based on the renormalized distribution, makes no assumptions concerning the discarded observations (beyond \pm 2.17 ). ML estimation based on the censored distribution assumes that the discarded observations are consistent in number with the $N(0,1)$ distribution. Since the area under the $N(0,1)$ curve is about $0.015(>2.17)$ and $0.015(<-2.17)$, censoring assumes about 15 censored observations at each end of the distribution $(0.015$ $\times 1,000)$. Note also that the observations are assumed to be consistent in number with the specified distribution but need not be consistent with respect to their position on the real line. In view of this assumption of consistency in number, Ulrich and Miller (1994) expressly presented censoring as an approximate procedure. Clearly, there is no compelling reason that outlying observations, owing to an extraneous process, should be consistent in number with the target distribution. This manner of accommodating outlying observations does allow one to carry out a post hoc examination of excluded observations - that is, one can calculate the exceedence probability of an excluded observation. Note that the information required to calculate these probabilities is part of the required information (Table 1) —namely, the cumulative distribution functions. As we will illustrate below, one can thus arrive at a posterior quantification of the degree to which observations are outlying. In contrast to censoring, truncation involves the discarding of data beyond given points and a renormalization of the distribution function. No assumptions are made concerning the discarded data points. As such, this procedure is superior to censoring in theory, but estimation subject to truncation is computationally more difficult (Ulrich \& Miller, 1994) and often results in less stable estimates.

As is clear in Tables 2D and 2E, our approach to censoring and truncation in the case of a mixture distribution involves the standard correction of the mixture distribution function and the associated loglikelihood function. 
This procedure does not take into account the fact that outliers may occur only in (say) one of the component distributions. For instance, imagine a mixture consisting of a fast and a slow component distribution, both relating to target processes. A subject will produce RTs belonging to the slow or to the fast component. Furthermore, imagine that slow outliers, owing to an extraneous process, are generated only when subjects produce fast RTs (i.e., belonging to the fast component in the mixture). In this situation, slow outliers will not necessarily be visible. In fitting the mixture, we imagine that the outlying RTs do little harm, since they will be indistinguishable from the slow RTsthat is, they will not bias estimates of the parameters of the fast component. Rather, they will be assigned to the slow component, where (it is hoped) they will blend in. The same applies to fast outliers produced only in the slow component. These will be indistinguishable from the RTs of the fast component.

\section{Multicase Analysis}

So far, we have considered RT data of a single subject. It is convenient, in an experimental design, to calculate required parameter estimates for each subject within each experimental condition separately. The estimates can subsequently be analyzed in the general linear model. However, the present approach does lend itself readily to multicase analyses. This simply involves the summation, over cases, of loglikelihood function values. For instance, one may want to assess the effect of a drug on the individual parameters of an RT distribution in a single subject. Suppose one has obtained RTs on a given task before and after administering the drug. In a multicase analysis, one can fit the RT distributions to the two data sets in two stages: one without any constraints on the parameters and one with equality constraints on (all or some) of the parameters. The difference in loglikelihood can then be used to test the hypothesis that the drug has no effect on the parameter values.

In multicase analyses, the specified distribution and the presence and degree of truncation or censoring may vary over the cases. For instance, in RT research, an experimental design may yield data from a mixture distribution, as well as data sampled independently from the component distributions. Multicase analyses can be used to investigate whether the component distributions are the same as those of the mixture (see Yantis et al., 1991). If RTs are collected in blocks, multicase analyses may be used to investigate the changes, if any, in the characteristics of the RT distributions over the blocks. Multicase analyses thus provide a convenient method to assess parameter variability, or drift (Burbeck \& Luce, 1982; Van Zandt \& Ratcliff, 1995). This method is, however, crude in that it is limited to modeling discrete, rather than continuous, changes in parameters.

\section{Loglikelihood Difference Test}

As was mentioned, we may impose equality constraints (within a case or across cases in multicase analyses) on the estimated parameters or fix parameters to given values. The latter allows us to estimate parameters of special cases of distributions. For instance, fixing the shape parameter $(\gamma)$ to equal 2 in the Weibull results in the so-called Rayleigh distribution(Johnson et al., 1994). The tenability of restrictions, both equality constraints and fixed parameters, may be evaluated by means of the LD test - that is, minus twice the difference in loglikelihoods of the model with and the model without the restrictions (Azzelini, 1996). Under the null hypothesis (i.e., restrictions are true), this difference is asymptotically $\chi^{2}$ distributed with degrees of freedom $(d f)$ equaling the difference in the number of parameters between the models with and without the constraints. Using the LD test, one thus can carry out detailed comparisons of RT distributions observed in difference cases or under different conditions. In addition to the LD $\chi^{2}$ test, the standard error of the estimate may be employed to test whether a given parameter deviates significantly from a given value (Azzelini, 1996). The LD test will be used below in the three real-data illustrations.

\section{Bounds or Box Constraints}

As we have seen above, the parameters of many distributions are bounded. For instance, the scale and shape parameters of the Weibull and gamma distributions are greater than zero. NPSOL includes the facility to impose bounds on the parameters, so that such constraints can be accommodated readily. The specification of bounds is useful, since limiting the parameter space often facilitates optimization.

\section{Limitations of ML}

The present framework is based solely on ML estimation. As was mentioned above, ML estimation has the advantage that under certain conditions, the estimates have desirable characteristic, such as maximum efficiency, consistency, and asymptotic unbiasedness (Miller \& Miller, 1999). Also, ML is closely associated with LD tests and various goodness-of-fit criteria, which may be used to evaluate and compare models. These include socalled information criteria, such as the Akaike information criterion (AIC; Akaike, 1974) and the Bayesian information criterion (BIC; Schwarz, 1978).

However, as has been noted by Heathcote (1996) and Van Zandt (2000) in the context of distributional analyses, the conditions may not be satisfied in the case of the some of the distributions considered above or in the case of mixtures of these distributions. Some problematic situations are well known. For instance, Johnson et al. (1994, p. 656) pointed out that in the Weibull distribution, ML estimates are "regular" (i.e., they satisfy the conditions and display expected asymptotic characteristics) when the parameter $\gamma$ is larger than 2 . In the case of the log-normal distribution, Johnson et al. (1994, p. 224) pointed out that the ML function tends to infinity as the shift parameter $\xi$ approaches the minimum observed RT. In the case of a mixture distribution, similar problems may emerge (Everitt \& Hand, 1981). For instance, Everitt and Hand discussed the fact that the LD test cannot be used to test the 
number of components in a mixture (e.g., two vs. one component). These problems do not generally render ML estimation unfeasible. They do mean that one should consider ML solutions carefully and take note of the situations in which regularity conditions are possibly violated (see Johnson et al., 1994). The parameter estimates should be least be admissible and interpretable, the standard errors should be small relative to the estimates, and the model should fit reasonably well. We use the Pearson $\chi^{2}$ as a goodness-of-fit measure (Greenwood \& Nikulin, 1996).

Below, we will present three small simulation studies and three illustrative analyses. The simulations are limited in scope. They are presented to demonstrate that, given modest sample sizes, the present approach works acceptably well in terms of parameter recovery and accuracy of standard errors. We limit our attention to estimation with and without censoring. Truncation requires large sample sizes to work well (Ulrich \& Miller, 1994). The three illustrative analyses concern real data. Since these are presented merely to demonstrate some possibilities offered by the present approach, we will not discuss substantive issues. Parameters were estimated with a Digital Visual FORTRAN 77 program. All analyses were carried out on a 500-MHz Pentium III computer.

\section{THREE SMALL SIMULATION STUDIES}

\section{Simulation 1: Single-Component Distributions Subject to Censoring}

The first simulation will address the success with which we can recover parameters in the case of the log- normal, ex-Gaussian, Weibull, gamma, and Wald distributions. This is a partial replication of Van Zandt's (2000) study. However, here, we also will compare standard errors based on the observed information matrix with the observed variability of the parameters, and we will fit the models with and without censoring. Finally, we will include the log-normal distribution.

We simulated 1,000 samples of 250 RTs distributed according to the distributions mentioned. In the case of the Weibull, the Wald, the gamma, and the ex-Gaussian distributions, we used the same parameter values as Van Zandt (2000; see Table 3 ). In the case of the log-normal, we choose the following parameter values: $\mu=5.187, \sigma=$ $0.472, \xi=600$. Each set of parameter values gives rise to RTs with an expected mean of 800 and a standard deviation of 100 . In fitting the models subject to censoring, we discarded the slowest 10 and the fastest 25 RTs- that is, $14 \%$ of the data. No outlying RTs were added, so here we satisfied the assumption that the censored RTs are compatible with the fitted distribution. Table 3 contains the true parameter values, the means $(M)$ and standard deviations $(S D)$ of the estimated parameters, and the means of the standard errors $\left(M S_{\mathrm{e}}\right)$. If the standard errors are accurate, these should resemble the standard deviations of the estimated parameters.

The results in Table 3 show that the parameters are recovered well when no censoring is imposed. There is some evidence of bias, since two-sided $t$ tests revealed that the mean parameter estimates of the Weibull, the Wald, and the gamma distributions (see italicized mean parameter estimates) deviated somewhat from their expected values,

Table 3

Results of Fitting Distributions to Simulated Data With and Without Censoring, Based on 1,000 Replications, Each Comprising 250 Observations

\begin{tabular}{|c|c|c|c|c|c|c|c|c|c|c|}
\hline Weibull & $\alpha$ & $\gamma$ & $\xi$ & $S D(\alpha)$ & $M S_{\mathrm{e}}(\alpha)$ & $S D(\gamma)$ & $M S_{\mathrm{e}}(\gamma)$ & $S D(\xi)$ & $M S_{\mathrm{e}}(\xi)$ & Fail \\
\hline True & 163.3 & 1.50 & 652.7 & - & - & - & - & - & - & - \\
\hline Mean 1 & 160.7 & 1.48 & 654.5 & 8.07 & 8.04 & 0.088 & 0.086 & 2.83 & 2.26 & 11 \\
\hline Mean2 & 164.3 & 1.53 & 651.7 & 10.08 & 12.97 & 0.131 & 0.161 & 5.82 & 8.68 & 1 \\
\hline Log-Normal & $\mu$ & $\sigma$ & $\xi$ & $S D(\mu)$ & $M S_{\mathrm{e}}(\mu)$ & $S D(\sigma)$ & $M S_{\mathrm{e}}(\sigma)$ & $S D(\xi)$ & $M S_{\mathrm{e}}(\xi)$ & Fail \\
\hline True & 5.187 & .472 & 600.0 & - & - & - & - & - & - & - \\
\hline Mean 1 & 5.178 & .476 & 600.5 & 0.108 & 0.114 & 0.054 & 0.056 & 17.23 & 17.81 & 13 \\
\hline Mean2 & 5.192 & .475 & 596.3 & 0.172 & 0.173 & 0.084 & 0.084 & 30.35 & 29.85 & 18 \\
\hline Wald & $\kappa$ & $\lambda$ & $\xi$ & $S D(\kappa)$ & $M S_{\mathrm{e}}(\kappa)$ & $S D(\lambda)$ & $M S_{\mathrm{e}}(\lambda)$ & $S D(\xi)$ & $M S_{\mathrm{e}}(\xi)$ & Fail \\
\hline True & 200.0 & 800.0 & 600.0 & - & - & - & - & - & - & - \\
\hline Mean 1 & 199.1 & 844.6 & 601.2 & 17.3 & 17.0 & 270.1 & 267.2 & 16.1 & 15.7 & 2 \\
\hline Mean2 & 198.9 & 850.0 & 601.7 & 21.7 & 23.4 & 341.0 & 386.1 & 21.3 & 23.4 & 50 \\
\hline Gamma & $\beta$ & $\alpha$ & $\xi$ & $S D(\beta)$ & $M S_{\mathrm{e}}(\beta)$ & $S D(\alpha)$ & $M S_{\mathrm{e}}(\alpha)$ & $S D(\xi)$ & $M S_{\mathrm{e}}(\xi)$ & Fail \\
\hline True & 4.00 & 5.0 & 600.0 & - & - & - & - & - & - & - \\
\hline Mean 1 & 3.97 & 51.5 & 602.7 & 1.04 & 0.961 & 8.00 & 7.96 & 18.5 & 17.3 & 0 \\
\hline Mean2 & 4.28 & 50.7 & 597.9 & 1.71 & 1.890 & 10.30 & 12.50 & 30.1 & 35.2 & 13 \\
\hline Ex-Gaussian & $\mu$ & $\sigma$ & $\tau$ & $S D(\mu)$ & $M S_{\mathrm{e}}(\mu)$ & $S D(\sigma)$ & $M S_{\mathrm{e}}(\sigma)$ & $S D(\tau)$ & $M S_{\mathrm{e}}(\tau)$ & Fail \\
\hline True & 705.1 & 31.6 & 94.8 & - & - & - & - & - & - & - \\
\hline Mean 1 & 705.8 & 31.6 & 94.4 & 5.92 & 5.70 & 4.69 & 4.50 & 8.16 & 8.03 & 0 \\
\hline Mean2 & 705.9 & 31.4 & 94.3 & 6.41 & 6.11 & 5.90 & 5.49 & 9.33 & 9.07 & 0 \\
\hline
\end{tabular}

Note-Censoring was limited to the top 25 and bottom 10 observations (i.e., $14 \%$ of the data). Italic mean values differ significantly from the true values ( $t$ test, expected Type I error rate $\alpha=.0005$, two-sided). Fail is the number of failed analyses of the total of $N=1,000$. Mean2 and mean 1 are the mean estimates with and without censoring, respectively. $S D$ and $M S_{\mathrm{e}}$ are the standard deviation of the estimates and the mean standard error, respectively. 
given an expected Type I error rate, $\alpha$, of .0005. Since the sample size of about 1,000 confers great power for detecting minor differences, we do not consider these to be serious. The same applies to the estimates obtained after the imposition of censoring. Note that the estimate of the parameter $\lambda$ of the Wald appears to be greatly biased. Van Zandt (2000) observes the same effect. However, the standard deviation is such that this effect, although systematic, cannot be judged to be too serious.

Without censoring, the mean standard errors and the standard deviations of the estimates agreed well. With censoring, the mean standard errors were clearly larger than the standard deviation of the parameter estimates, in the case of the Weibull, the Wald, and the gamma distributions. This means that the standard error underestimated the precision of the point estimate. The differences were not such that the use of standard errors should be abandoned. However, in the case of censoring, they should be viewed as approximate. Overall, the number of failed analyses was very small (in all cases, failure meant that the minus the information matrix was not positive definite).

Although the present simulation was limited to censoring, we did attempt to repeat the analyses subject to truncation. With the exception of the ex-Gaussian, the results were very poor, for reasons we do not understand (but see Ulrich \& Miller, 1994,p. 69). For instance, very often the shift parameter (Weibull, log-normal, Wald, gamma) hit the lower bound. This bound was chosen very liberally. This, in turn, affected the other parameter estimates, which, like the shift parameter, displayed bimodal histograms. Carrying out the analyses with the shift parameters fixed to the true values greatly alleviated these problems. In contrast, truncation in the ex-Gaussian did not result in appreciable problems. Here 18 of the 1,000 analyses failed. The results, which may be compared with those in Table 3, are the following: $M(\mu)=703.9, S D(\mu)=$ $10.2, M S_{\mathrm{e}}(\mu)=10.9, M(\sigma)=31.1, S D(\sigma)=12.4$, $M S_{\mathrm{e}}(\sigma)=14.2, M(\tau)=96.3, S D(\tau)=23.8, M S_{\mathrm{e}}(\tau)=$ 26.0. Note that, given truncation, the variability of the estimates is much larger than in the case of censoring.

\section{Simulation 2: Simultaneous Analyses of a Single-Component Ex-Gaussian and a Mixture of Ex-Gaussians}

The second simulation study was limited to the exGaussian distribution. Here, we simulated data in two conditions. In Condition 1, the data consisted of 100 RTs, distributed according to the ex-Gaussian with the following parameter values: $\mu_{1}=705.1, \sigma_{1}=31.6$, and $\tau_{1}=$ 94.8. In Condition 2, the data consisted of 400 RTs distributed according to a two-component mixture of exGaussians. In the first component, consisting of $150 \mathrm{RTs}$, the parameters equaled those in Condition 1 -that is, $\mu_{1}=705.1, \sigma_{1}=31.6$, and $\tau_{1}=94.8$. In the second component, consisited of $250 \mathrm{RTs}$, the parameters equaled $\mu_{2}=605.1, \sigma_{2}=31.6$, and $\tau_{2}=84.8$. The mixing proportion, $p_{1}$, equaled $150 / 400=.375$ (the mixing proportion, $p_{2}$, equals $\left.1-p_{1}\right)$. This model may obtain when a subject responds according to Strategy A in Condition 1 and according to either Strategy A or B in Condition 2. In Condition 2, the subject responds according to Strategy A with a probability of .375 .

This simulation illustrates some of the possibilities offered by the present framework. We fitted the following two-case model. The first case was the single-component ex-Gaussian $(N=100)$. The second case was the mixture of two ex-Gaussians $(N=400)$. We constrained the parameters of the first component to be equal to the parameters estimated in the first case. In addition, we fitted the model with and without censoring. In the case of censoring, we removed the 10 largest RTs in Case 1 and the 20 fastest and 20 slowest RTs in Case 2. Including the mixing proportion, we estimated seven parameters. We carried out a total of 500 replications.

Table 4 contains the results. Without censoring, $5 \%$ of the analyses failed (minus information matrix not positive definite). The parameters were recovered well, although $t$ tests $(\alpha=.0005$, two-sided) indicated some bias in the parameters $\mu_{1}, \tau_{1}$, and $\mu_{2}$. With censoring, $11 \%$ of the analyses failed. Here, we observe some bias in the parameters $\mu_{1}$, $\tau_{1}$, and $\mu_{2}$. In addition, we observe a slight bias in the estimate of the mixing proportion. Clearly, as in the previous simulation, the bias was quite small. Overall, the agreement between the mean standard errors and the standard deviations of the estimates was considered to be acceptable.

\section{Simulation 3: Fitting the Weibull Distribution to Ex-Gaussian Distributed Data}

Logan's instance theory of automaticity was based on a model involving single-step direct retrieval from memory of prior solutions to a given problem. Logan suggested that under the assumptions of his model, retrieval times, and so RTs, are asymptotically Weibull distributed (Logan, 1992; see Colonius, 1995, for more a more exact statement of conditions in which the Weibull will hold). Since it is gen-

Table 4

Results of Fitting a Two-Case Model

\begin{tabular}{|c|c|c|c|c|c|c|c|}
\hline \multirow[b]{2}{*}{ Measure } & \multicolumn{3}{|c|}{ Component 1} & \multirow[b]{2}{*}{$p_{1}$} & \multicolumn{3}{|c|}{ Component 2} \\
\hline & $\mu_{1}$ & $\sigma_{1}$ & $\tau_{1}$ & & $\mu_{2}$ & $\sigma_{2}$ & $\tau_{2}$ \\
\hline \multicolumn{8}{|c|}{ No Censoring $\left(\right.$ Fail $\left.=26^{*}\right)$} \\
\hline True & 705.10 & 31.60 & 94.80 & .375 & 605.10 & 31.60 & 84.8 \\
\hline Mean $\dagger$ & 706.60 & 31.20 & 91.50 & .361 & 606.80 & 31.90 & 84.7 \\
\hline$S D \dagger$ & 7.62 & 6.26 & 10.03 & .107 & 7.98 & 5.35 & 21.4 \\
\hline$M S_{\mathrm{e}} \ddagger$ & 7.54 & 6.14 & 10.09 & .114 & 8.60 & 5.35 & 23.7 \\
\hline \multicolumn{8}{|c|}{ Censored $\left(\right.$ Fail $\left.=55^{*}\right)$} \\
\hline True & 705.10 & 31.60 & 94.80 & .375 & 605.10 & 31.60 & 84.8 \\
\hline Mean $\dagger$ & 706.90 & 30.57 & 91.36 & .354 & 607.50 & 32.00 & 85.3 \\
\hline$S D \dagger$ & 7.97 & 8.87 & 10.78 & .111 & 9.09 & 7.38 & 23.1 \\
\hline$M S_{\mathrm{e}} \ddagger$ & 7.68 & 8.57 & 10.94 & .125 & 9.95 & 7.83 & 25.3 \\
\hline
\end{tabular}

Note-Case 1 is a simple ex-Gaussian; Case 2 is a constrained mixture of ex-Gaussians. Results are based on 500 replications, each comprising 100 (Case 1) and 400 (Case 2) observations. In Case 1, the 10 largest observations were censored (10\% of the data); in Case 2, the 20 largest and 20 slowest ( $10 \%$ of the data) were censored. *Number of failed analysis of the total of 500. † Mean and standard deviation of estimated parameter. Italic mean values differ significantly from the true values ( $t$ test, $\alpha=.0005$, two-sided). $\quad{ }^{\ddagger} M S_{\mathrm{e}}$ is the mean standard error of the parameter estimate. 
erally accepted that the ex-Gaussian distribution fits RT data well, Logan investigated whether the Weibull distribution fits data that are ex-Gaussian distributed. We repeated a part of his simulation. Our objectives were (1) to investigate the performance of the Pearson $\chi^{2}$ goodness-of-fit test ${ }^{2}$, and (2) to check the goodness of fit of the Weibull, using the Pearson $\chi^{2}$ test. We considered four of Logan's eight parameter sets. We generated data distributed according to an ex-Gaussian with the following parameter values: (1) $\mu=400, \sigma=50, \tau=$ 100; (2) $\mu=400, \sigma=50, \tau=200$; (3) $\mu=400, \sigma=$ $100, \tau=100$; (4) $\mu=400, \sigma=100, \tau=200$. Given each set of parameters, we generated 400 data sets, each comprising 200 observations, a total of $4 \times 400 \times 200$ observations. The probability of generating negative RTs was not a concern, because the probability of $x<0$ was largest in Parameter Set 3 but here was only about 6E-06 (in fact, a single negative value was detected and replaced). We eschewed Logan's other parameter sets (same values of $\sigma$ and $\tau$, but $\mu$ equal to 200), because here the probability equaled about .0063 in the worst casethat is, $\sigma=100, \tau=100$. We fitted the ex-Gaussian and the Weibull to each of the 400 data sets. Goodness-of-fit tests were carried by creating 20 equiprobable bins and comparing the observed and the expected bin counts by means of Pearson $\chi^{2}$ tests (see Note 2). The choice of 20 bins was arbitrary (see Heathcote, 1996, for a rule of thumb relating to the number of bins).

The results, based on 400 replications are shown in Table 5A. These results include the mean parameter estimates, the standard deviation of the parameter estimates, and the mean standard errors. The number of failed analyses is also reported (minus information matrix not positive definite).
The results in Table 5A show that the parameters of the ex-Gaussian were successfully recovered. The standard deviations of the parameter estimates resembled the mean standard error. In addition, the Pearson $\chi^{2}$ test performed reasonably well. Naively treating the test statistic as $\chi^{2}$ distributed with $16 d f$ s (see Note 2 ) and rejecting the nullhypothesis of the ex-Gaussian if the test statistic exceeded $26.3(\alpha=.05)$ resulted in the incorrect rejection of the hypothesis in $3.5 \%, 5.7 \%, 6.0 \%$, and $6.0 \%$ of the cases. The number of failed analyses was small (between 0 and $2 \%$ ).

The results relating to the Weibull distribution are surprising. Given the Type I error rate, $\alpha$, of .05, the Pearson $\chi^{2}$ test resulted in a rejection of the hypothesis that the data are distributed according to the Weibull distribution in $48.2 \%, 57.2 \%, 19.0 \%$, and $51.0 \%$ of the cases. The mean and standard deviations of the Pearson $\chi^{2}$ statistics clearly exceeded the naive expectation of 16 and a standard deviation of $\sqrt{(2 \times 16)}$ - that is, 5.657 ( $d f$ and $2 \times d f$ are the mean and variance of the central $\chi^{2}$ distribution). Another striking result was the difference between the standard deviations of the parameter estimates and the mean standard errors, based on the observed information matrix. The mean standard errors greatly underestimated the actual variability of the estimates. Visual inspection of the histograms of the Weibull parameter estimates indicated that the greater standard deviations of the estimates were not due to outlying observations. Generally, the interpretation of standard errors is valid only if the model fits the data and distributional assumptions are satisfied. Here, the misfit of the Weibull distribution may have resulted in quite unreliable standard errors.

On the basis of the fits of the Weibull to the ex-Gaussian data, we are inclined to conclude that the Weibull does not

Table 5A

Results of Fitting the Weibull to Ex-Gaussian Data

\begin{tabular}{|c|c|c|c|c|c|c|c|c|c|c|c|c|}
\hline \multirow[b]{2}{*}{ Measure } & \multicolumn{6}{|c|}{ Ex-Gaussian (True Model) } & \multicolumn{6}{|c|}{ Weibull } \\
\hline & $\mu$ & $\sigma$ & $\tau$ & $\chi^{2}(16)$ & $\%$ reje & Faild $^{\mathrm{d}}$ & $\alpha$ & $\gamma$ & $\xi$ & $\chi^{2}(16)$ & $\%$ rej & Fail \\
\hline True & 400.00 & 50.00 & 100.0 & - & - & - & - & - & - & - & - & - \\
\hline Mean ${ }^{\mathrm{a}}$ & 399.50 & 48.90 & 99.9 & 16.1 & 3.5 & 0 & 232.8 & 1.960 & 292.80 & 27.3 & 48.2 & 2 \\
\hline$S D^{\mathrm{b}}$ & 9.11 & 6.83 & 11.1 & 5.3 & - & - & 27.2 & 0.237 & 27.20 & 9.3 & - & - \\
\hline$M S_{\mathrm{e}}^{\mathrm{c}}$ & 9.03 & 6.75 & 10.9 & - & - & - & 10.7 & 0.118 & 4.82 & - & - & - \\
\hline True & 400.0 & 50.00 & 200.0 & - & - & - & - & - & - & - & - & - \\
\hline Mean & 400.8 & 49.70 & 198.2 & 16.9 & 5.7 & 0 & 327.3 & 1.550 & 305.10 & 29.5 & 57.2 & 0 \\
\hline$S D$ & 11.5 & 9.14 & 17.7 & 5.7 & - & - & 32.0 & 0.163 & 24.30 & 10.1 & - & - \\
\hline$M S_{\mathrm{e}}$ & 11.1 & 9.08 & 17.6 & - & - & - & 16.6 & 0.086 & 3.37 & - & - & - \\
\hline True & 400.0 & 100.0 & 100.0 & - & - & - & - & - & - & - & - & - \\
\hline Mean & 401.1 & 100.4 & 97.6 & 16.2 & 6.0 & 1 & 391.1 & 2.62 & 150.8 & 21.7 & 19.0 & 8 \\
\hline$S D$ & 17.9 & 11.2 & 18.2 & 5.5 & - & - & 48.4 & 0.38 & 44.9 & 7.0 & - & - \\
\hline$M S_{\mathrm{e}}$ & 17.7 & 10.9 & 17.8 & - & - & - & 23.9 & 0.21 & 18.8 & - & - & - \\
\hline True & 400.0 & 100.0 & 200.0 & - & - & - & - & - & - & - & - & - \\
\hline Mean & 401.0 & 99.2 & 198.7 & 16.4 & 6.0 & 1 & 468.7 & 1.970 & 183.60 & 27.9 & 51.3 & 0 \\
\hline$S D$ & 18.5 & 14.5 & 22.2 & 5.9 & - & - & 54.8 & 0.249 & 47.70 & 9.6 & - & - \\
\hline$M S_{\mathrm{e}}$ & 18.1 & 13.4 & 21.8 & - & - & - & 21.7 & 0.119 & 9.82 & - & - & - \\
\hline
\end{tabular}

Note-The results are based on 400 replications; each replication comprises 200 observations aThe mean estimate. bThe standard deviation of the estimate. ${ }^{\mathrm{c}}$ Mean standard error. dThe number of failed analyses (information matrix not positive definite) of the total of 400 . eFor the true model, the expected number of rejections (i.e., Type I error) is $5 \%$ (this expectation is based on the assumption that the Pearson $\chi^{2}$ test statistic is $\chi^{2}(16)$ distributed; see Note 2$)$. 
fit ex-Gaussian-generated data very well. However, it appears to be more relevant to investigate whether the exGaussian fits Weibull-generated data, given that RTs are Weibull distributed ex hypothesi. In addition, Johnson et al., (1994, p. 656) pointed out that ML estimates of the Weibull are regular, in the sense that the sampling distributuion of the extimates is asymptotically normal only if the parameter $\gamma$ is greater than about 2 . In Table $5 \mathrm{~A}$, where mean $[\gamma]=1.55$, this is not the case, so that the discrepancy between the mean standard errors and the standard deviation of estimates may be due to a lack of regularity. We reversed the procedure by simulating data according to the Weibull distribution and fitting the ex-Gaussian. Again, we simulated 400 sets comprising 200 observations, given each of the following four sets of parameters: (1) $\alpha=235, \gamma=2, \xi=290$; (2) $\alpha=330, \gamma=1.54, \xi=$ 303; (3) $\alpha=295, \gamma=2.6, \xi=148$; (4) $\alpha=470, \gamma=2$, $\xi=182$. The results are shown in Table 5B. The ML estimates of the parameters were slightly biased, but the agreement between the standard deviations of the parameter estimates and the mean standard errors was acceptable, both in the $\gamma=1.54$ and in the other conditions. The Weibull distribution (the true model) was rejected in $6 \%, 9 \%, 7 \%$, and $6 \%$ of the replications, given the $\alpha$ of .05. Turning to the results relating to the ex-Gaussian, we find that the number of rejections was a lot lower that those observed above: $14 \%, 22 \%, 8 \%$, and $15 \%$. The rejection rate of $8 \%$ seems quite low, but the results obtained with the third parameter set may not be reliable. Here, $12.3 \%$ of the analyses failed (elsewhere in Table 5B, the percentage is no greater than $0.5 \%$ ). Nonetheless, given the chosen parameter values, we observed a clear asymmetry, in that the ex-Gaussian fitted Weibull-generated data more readily than the Weibull fitted ex-Gaussian-generated data. Although there were still notable discrepancies between the standard deviations and the mean standard er- rors of the ex-Gaussian parameters, these discrepancies were much smaller than those in Table 5A.

On the basis of the present results, given the chosen parameter values, we may conclude that ML is quite successful in recovering the true parameter values (see Van Zandt, 2000). The standard errors are sufficiently accurate, at least, to provide a good indication of the curvature of the loglikelihood function. We now will present three analyses of real data. We emphasize that we present these mainly to illustrate some of the possibilities of the present framework.

\section{THREE REAL DATA ANALYSES}

\section{Simple Two-Choice RT Task With and Without Stopping}

This data set consists of simple two-choice RT data obtained in two conditions. In the first (standard) condition, the subject was presented with a visual warning signal followed by an imperative signal. The imperative signal was an arrow presented on a computer monitor, pointing either to the left or to the right. If the arrow pointed to the left (right), the subject was required to press a button with the index finger of the right (left) hand, to the right (left) on a computer keyboard. In the second (stop) condition, the task was identical, but at some time following the warning signal, a visual stop signal would appear in some, but not all, trials. Following the stop signal, the subject was required to inhibit his (her) response - that is, do nothing. The timing of the stop signal was such that the subject could successfully inhibit about $50 \%$ of the responses. The objective of this task was to assess the subject's ability to inhibit a response (see Logan \& Cowan, 1984). The responsestimulus interval (RSI) was randomly chosen from five intervals. We were interested in whether the RTs in the first condition would be comparable to those in the second condition, when no stop signal was presented. In all, 196

Table 5B

Results of Fitting the Weibull to Ex-Gaussian Data

\begin{tabular}{|c|c|c|c|c|c|c|c|c|c|c|c|c|}
\hline \multirow[b]{2}{*}{ Measure } & \multicolumn{6}{|c|}{ Ex-Gaussian } & \multicolumn{6}{|c|}{ Weibull (True Model) } \\
\hline & $\mu$ & $\sigma$ & $\tau$ & $\chi^{2}(16)$ & \%rej & Fail & $\alpha$ & $\gamma$ & $\xi$ & $\chi^{2}(16)$ & \%rej & Fail \\
\hline True & - & - & - & - & - & - & 235.0 & 2.000 & 290.00 & - & - & - \\
\hline Mean & 412.6 & 69.8 & 85.7 & 19.6 & 14 & 2 & 229.9 & 1.960 & 294.20 & 17.1 & 6 & 0 \\
\hline$S D$ & 17.6 & 10.8 & 15.6 & 6.7 & - & - & 13.8 & 0.161 & 9.23 & 5.2 & - & - \\
\hline$M S_{\mathrm{e}}$ & 15.4 & 10.6 & 15.9 & - & - & - & 14.3 & 0.163 & 9.24 & - & - & - \\
\hline True & - & - & - & - & - & - & 330.0 & 1.540 & 304.00 & - & - & - \\
\hline Mean & 394.0 & 64.5 & 207.3 & 21.4 & 22 & 0 & 323.5 & 1.510 & 308.70 & 17.30 & 9 & 0 \\
\hline$S D$ & 27.2 & 21.4 & 25.6 & 7.3 & - & - & 19.0 & 0.108 & 7.02 & 6.19 & - & - \\
\hline$M S_{\mathrm{e}}$ & 17.9 & 15.4 & 23.0 & - & - & - & 18.9 & 0.102 & 5.86 & - & - & - \\
\hline True & - & - & - & - & - & - & 395.0 & 2.600 & 148.0 & - & - & - \\
\hline Mean & 416.9 & 119.2 & 80.9 & 17.4 & 8 & 49 & 385.8 & 2.550 & 155.5 & 16.80 & 7 & 0 \\
\hline$S D$ & 22.9 & 12.3 & 20.4 & 6.0 & - & - & 32.5 & 0.280 & 27.4 & 5.98 & - & - \\
\hline$M S_{\mathrm{e}}$ & 27.4 & 14.4 & 26.8 & - & - & - & 32.3 & 0.275 & 27.2 & - & - & - \\
\hline True & - & - & - & - & - & - & 470.0 & 2.000 & 182.0 & - & - & - \\
\hline Mean & 423.0 & 137.4 & 175.5 & 19.8 & 15 & 0 & 459.4 & 1.960 & 190.6 & 16.90 & 6 & 0 \\
\hline$S D$ & 35.8 & 22.4 & 32.1 & 6.6 & - & - & 29.5 & 0.161 & 19.3 & 5.65 & - & - \\
\hline$M S_{\mathrm{e}}$ & 30.7 & 21.4 & 31.8 & - & - & - & 28.2 & 0.161 & 17.9 & - & - & - \\
\hline
\end{tabular}

Note-The results are based on 400 replications; each replication comprises 200 observations (see Notes to Table 5A). 
RTs were recorded in the standard condition, and 126 RTs were recorded in the stop condition, in a single subject. Histograms of these RTs are shown in Figure 1. Since this analysis is illustrative, we fitted the ex-Gaussian, the log-normal, the gamma, the Wald, and the Weibull distributions to these data. We make no claims concerning the theoretical suitability of these distributions as models for two-choice RTs. The Wald is certainly unsuitable for twochoice RT data (Luce, 1986). First, we estimated the parameters without equality constraints. Subsequently, we introduced equality constraints and tested whether the distributions were identical across conditions. Table 6 contains the parameter estimates without equality constraints. It is striking that the standard errors of the shift parameters $(\xi)$ of the Wald and the log-normal were very large. Fixing these parameters to zero did not result in any appreciable change in loglikelihood [Wald, $\operatorname{LD} \chi^{2}(2)=1.0$, n.s., $\log$-normal, LD $\chi^{2}(2)=1.4$, n.s.].

To obtain an indication of goodness of fit, we created the arbitrary number of 11 equiprobable bins, using the ML estimates (see Heathcote, 1996, for a discussion of the choice of the number of bins), and we compared observed and expected bin counts by means of Pearson $\chi^{2}$ tests. Without equality constraints, we found the following values of the Pearson $\chi^{2}$ test statistics in the standard and the stop conditions: 12.9 and 11.9 (ex-Gaussian; $d f=7$ ), 17.9 and 10.0 (log-normal; $d f=7$ ), 16.0 and 7.1 (gamma; $d f=7$ ), 13.1 and 7.1 (Weibull; $d f=7$ ), and 7.3 and 8.9 (Wald; $d f=7$ ). It is striking that the five distributions fitted the observed data reasonably well (the critical value associated with $d f=7$ given $\alpha=.05$ was 14.06), even though the derivations of the distributions were dissimilar (see Van Zandt, 2000; Van Zandt \& Ratcliff, 1995). We used the LD $\chi^{2}$ test to test the hypothesis that the parameters of the specified distributions were equal. The observed ratios were $\chi^{2}$ distributed with $3 d f$, given the null hypothesis (identical distributions). These statistics ranged from 107 (ex-Gaussian) to 125 (Weibull), so we conclude that the distributions were not identical. The Pearson $\chi^{2}$ test also indicates the misfit. In the standard and the stop conditions, we observed the following test statistics: 44.5 and 75.1 (ex-Gaussian), 48.6 and 70.3 (lognormal), 53.6 and 70.6 (gamma), 69.4 and 62.5 (Weibull), and 49.0 and 68.8 (Wald). Clearly, for the present subject, the instruction to inhibit the reaction affected the distribution of the RTs, even when no stop signal was presented. Figure 1 shows the fitted densities of the gamma, ex-Gaussian, and log-normal superimposed on the histograms.

\section{XOR Two-Choice RT Data}

This data set was collected in a single subject during a two-choice RT task, the XOR task. The subject was seated in front of a monitor. Her hands rested on the table, with
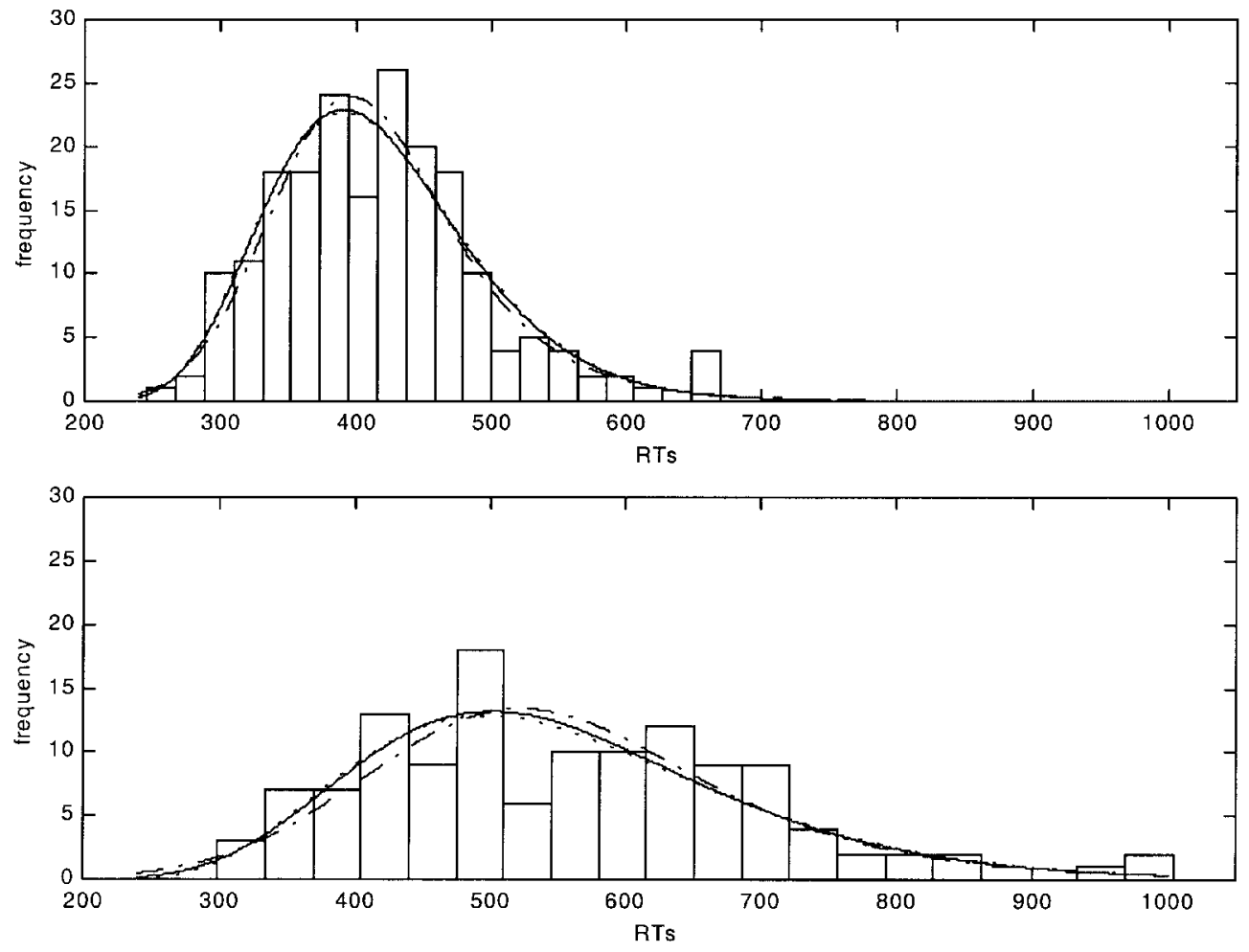

Figure 1. Illustration 1. Top: histogram of reaction times (RTs) obtained in simple two-choice RT task $(N=196)$. Bottom: histogram of RTs obtained using simple two-choice RT task including stop signal $(N=$ 126). Data shown were obtained in a trial without a stop signal. The fitted densities of the log-normal (solid line), the ex-Gaussian (dashed line), and the gamma (dotted line) distributions are included. 
Table 6

Illustration 1: Maximum Likelihood Parameter Estimates (Est) and Standard Errors (SEs)

\begin{tabular}{cccccccc}
\hline Distribution & \multicolumn{3}{c}{ Standard Condition } & & \multicolumn{3}{c}{ Stop Condition } \\
\cline { 3 - 4 } \cline { 6 - 8 } Ex-Gaussian & $\mu_{1}$ & $\sigma_{1}$ & $\tau_{1}$ & & $\mu_{2}$ & $\sigma_{2}$ & $\tau_{2}$ \\
\hline Est & 357.30 & 51.50 & 58.50 & & 451.8 & 98.5 & 103.9 \\
$S E$ & 8.49 & 5.56 & 8.71 & & 23.5 & 15.4 & 23.7 \\
Log-normal & $\mu_{1}$ & $\sigma_{1}$ & $\xi_{1}$ & $\mu_{2}$ & $\sigma_{2}$ & $\xi_{2}$ \\
\hline Est & 5.79 & 0.228 & $79.9^{*}$ & & 6.24 & 0.264 & $23.1^{*}$ \\
$S E$ & 0.25 & 0.060 & 79.5 & & 0.33 & 0.060 & 162.9 \\
Gamma & $\beta_{1}$ & $\alpha_{1}$ & $\xi_{1}$ & & $\beta_{2}$ & $\alpha_{2}$ & $\xi_{2}$ \\
\hline Est & 9.61 & 24.90 & 176.3 & & 5.94 & 58.9 & 205.7 \\
$S E$ & 4.28 & 6.33 & 48.2 & 3.16 & 18.7 & 79.6 \\
Weibull & $\gamma_{1}$ & $\alpha_{1}$ & $\xi_{1}$ & $\gamma_{2}$ & $\alpha_{2}$ & $\xi_{2}$ \\
\hline Est & 2.40 & 199.80 & 238.50 & & 2.01 & 308.1 & 282.5 \\
$S E$ & 0.16 & 9.90 & 6.79 & 0.21 & 23.7 & 15.8 \\
Wald & $\kappa_{1}$ & $\lambda_{1}$ & $\xi_{1}$ & $\kappa_{2}$ & $\lambda_{2}$ & $\xi_{2}$ \\
\hline Est & 341.2 & $6,642.6$ & $74.5^{*}$ & 527.0 & $7,143.7$ & $28.6^{*}$ \\
$S E$ & 78.2 & $4,837.7$ & 78.0 & 150.5 & $6,609.6$ & 149.9 \\
\hline
\end{tabular}

Note-Histograms of RTs are displayed in Figure 1. Parameters with subscript 1 were estimated using $N=196$ data obtained in the standard condition (see Figure 1, top). Parameters with subscript 2 were estimated using $N=126$ data obtained in the stop condition (see Figure 1, bottom). No equalities were imposed. Fitted densities were superimposed on the histograms in Figure 1. *These shift parameters do not differ significantly from zero. Fixing both to zero resulted in an LD $\chi^{2}$ of $1.4(d f=2$, n.s. $)$ in the log-normal and an $\operatorname{LD} \chi^{2}$ of $1.0(d f=2$, n.s. $)$ in the Wald.

a finger of each hand resting on designated keys of a standard keyboard. If two consonants or two vowels were presented, the subject was required to press a key on the left; otherwise, she pressed a key on the right. The RSI was distributed as $N(1500,300)$. The subject generated 500 responses, of which 41 involved errors of commission. Histograms of the data are shown in Figure 2. The histogram of the correct RTs has the characteristic positive skew and long tail. The 10 slowest RTs were 1,117 , $1,150,1,167,1,183,1,200,1,300,1,350,1,400,1,467$, and $1,517 \mathrm{msec}$. The histogram of the error RTs is concentrated at about $500 \mathrm{msec}$. The 5 slowest RTs were 567 , $583,600,900$, and $1367 \mathrm{msec}$. There were two apparently outlying error RTs (namely, 900 and 1,367 msec.).

We fitted the Weibull, gamma, ex-Gaussian, log-normal, and Wald distributions to the correct response RTs, subject to slight censoring and truncation to the right at 1,000 msec. We assessed goodness of fit by means of Pearson $\chi^{2}$ tests with nine equiprobable bins. The parameter estimates are shown in Table 7 . The Pearson $\chi^{2}$ tests indicated that the Weibull fit the data relatively poorly [Pearson $\chi^{2}(5)=$ 98.0]. The ex-Gaussian, gamma, log-normal, and the Wald all fitted about equally well (Pearson $\chi^{2}$ s with $5 d f \mathrm{~s}$ equal 25.6, 25.6, 21.8, and 23.2, respectively). These models do not fit by strict statistical standards (for instance, given an $\alpha=.01$ and $d f=5$, the critical value is 15.08). However, as was pointed out by Van Zandt (2000), large samples confer great power and make it almost impossible to obtain a well-fitting model using strict statistical criteria. This problem is well known in covariance structure modeling (e.g., Browne \& Cudeck, 1993; Jöreskog, 1993).
Since the Weibull fits relatively poorly and the Wald is unsuitable for two-choice RT data, we will report further results for the ex-Gaussian, log-normal, and gamma distributions. We calculated the exceedence probabilities of the 19 observations removed by censoring. The probabilities of the two slowest RTs $(1,517$ and $1,467 \mathrm{msec})$ were equal: .00094 and .0013 (ex-Gaussian), .00016 and .00027 (gamma), and .0015 and .0019 (log-normal). Since 1,517 and $1,467 \mathrm{msec}$ were the slowest of a total of $459 \mathrm{RTs}$, we consider these probabilities to be not particularly extreme.

We next investigated whether the distribution of the error RTs equaled that of the correct RTs. We limited our analyses to the ex-Gaussian, log-normal, and gamma distributions. We did not impose any censoring or truncation in the correct RT data, but we did impose censoring in the error RT data to accommodate the presence of the two slow RTs (900 and 1,367 msec). We first estimated the parameters without any equality constraints. Subsequently, we imposed equality constraints on the three parameters of the three distributions. LD $\chi^{2}$ tests indicated that these equality constraints were not tenable in any case: $\chi^{2}(3)=$ $29.06($ gamma $), \chi^{2}(3)=26.2(\log$-normal $)$, and $\chi^{2}(3)=$ 24.6 (ex-Gaussian). Inspection of the estimates revealed that the largest differences were observed with respect to the $\tau$ parameter in the ex-Gaussian distribution, the $\beta$ parameter in the gamma distribution, and the $\mu$ parameter in the log-normal distribution. For the sole purpose of illustrating the possibility of limiting equality constraints to particular parameters, we estimated these parameters $(\tau, \beta$, $\mu)$ without equality constraints, while retaining the other equality constraints. The results are shown in Table 8 . The 

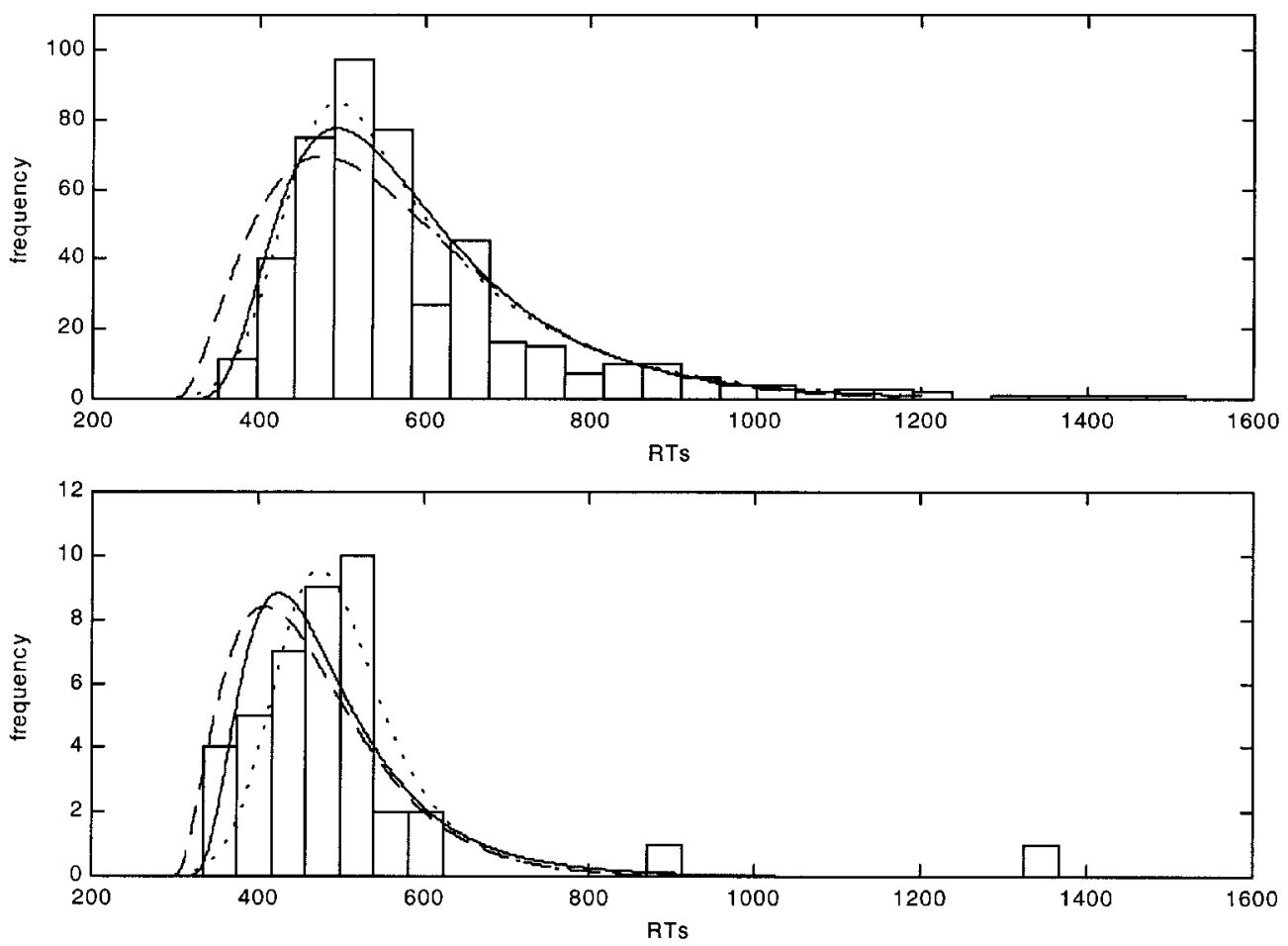

Figure 2. Illustration 2. XOR two-choice reaction time (RT) data (top, correct response RTs, $n=459$; bottom, error RTs, $n=41$ ). Superimposed are the fitted densities based on the parameter estimates in Table 8 . The solid line is the log-normal, the dotted line is the ex-Gaussian, and the dashed line is the gamma.

LD tests equaled LD $\chi^{2}(2)=7.2($ ex-Gaussian; $p=.03)$, $\mathrm{LD} \chi^{2}(2)=6.9(\mathrm{gamma} ;=.03)$, and $\operatorname{LD} \chi^{2}(2)=1.1$ $(\log$-normal; $p=.57)$. Figure 2 shows the fitted density function superimposed on the observed histograms. The exceedence probabilities of the excluded error RTs were $.00141(900 \mathrm{msec})$ and $.0000016(1,367 \mathrm{msec})$ in the exGaussian distribution. These probabilities suggested that the 1,367-msec RT was an outlier but that the $900-\mathrm{msec}$ RT was not. The same conclusion applied when the exceedence probabilities were calculated in the gamma and the log-normal distributions.

\section{Two-Choice RT Task Subject to Accuracy Requirements: Simultaneous Analysis of a Mixture of Ex-Gaussians and a Single-Component Ex-Gaussian}

This data set was obtained in a two-choice RT task in which the subject was required to respond at an average accuracy of $75 \%$ correct as quickly as possible. After each response, feedback about the current average accuracy was presented. Rewards were given after a series of stimuli. These series consisted of a number of stimuli (unknown to the subject) of between 11 and 35. Financial rewards were based on a ranking list that depended on accuracy scores (as close as possible to $75 \%$ correct). If the subjects scored equally with respect to accuracy, the reward then depended on the actual RT (the faster the RT, the greater the reward). The stimuli were two arrows presented on a computer monitor, one pointing to the left and one pointing to the right. Responding took place by pressing keys on the keyboard. The RSI was distributed as $N(1500,300)$. The data of 1 subject were analyzed. The goal of this experiment was to investigate the response process, given the accuracy requirement. We expected the RTs to display a mixture distribution, with a fast component (guessing) and a slow component (accurate responding). We expected the RTs of the error responses to display the same RT distribution as the fast component in the mixture. We expected $50 \%$ of the responses to belong to the fast (guessing) component and $50 \%$ to belong to the slow component. The slow component was expected to include no errors. We expected $50 \%$ of the responses belonging to the fast component to be errors.

Figure 3 displays the histograms of the RTs of the correct responses $(N=669)$ and of the RTs of the error responses $(N=233)$. We fitted a mixture of ex-Gaussian distributions to the correct response RTs and a single ex-Gaussian to the error response RTs. The decision to fit ex-Gaussians, rather than some other distribution, was arbitrary. However, mixture of the log-normal, Weibull, and gamma distributions cannot be fit in the present approach if the shift parameter varies over the components. This is due to the requirement in these distributions that $\xi<x$, where $\xi$ is the shift parameter. If the shift parameters vary over the com- 
Table 7

Illustration 2: Maximum Likelihood Estimates of Parameters of Five Distributions Fitted to XOR Task

\begin{tabular}{|c|c|c|c|c|c|c|}
\hline \multirow[b]{2}{*}{ Parameter } & \multicolumn{2}{|c|}{$\begin{array}{c}\text { Not Censored } \\
\text { or Truncated }\end{array}$} & \multicolumn{2}{|c|}{ Censored } & \multicolumn{2}{|c|}{ Truncated } \\
\hline & $M$ & $S E$ & $M$ & $S E$ & $M$ & $S E$ \\
\hline \multicolumn{7}{|l|}{ Weibull } \\
\hline$\alpha$ & 273.4 & 9.12 & 270.6 & 8.72 & 251.5 & 7.86 \\
\hline$\gamma$ & 1.52 & 0.053 & 1.59 & 0.061 & 1.83 & 0.077 \\
\hline$\xi$ & 348.6 & 1.385 & 348.2 & 1.70 & 346.5 & 2.77 \\
\hline \multicolumn{7}{|l|}{ Ex-Gaussian } \\
\hline$\mu$ & 437.5 & 5.64 & 438.2 & 5.72 & 444.3 & 6.23 \\
\hline$\sigma$ & 44.86 & 4.24 & 45.1 & 4.27 & 47.6 & 4.48 \\
\hline$\tau$ & 156.0 & 8.97 & 154.0 & 9.20 & 132.8 & 10.64 \\
\hline \multicolumn{7}{|l|}{ Gamma } \\
\hline$\beta$ & 2.54 & 0.211 & 2.69 & 0.246 & 3.61 & 0.48 \\
\hline$\alpha$ & 98.73 & 7.65 & 92.62 & 7.72 & 66.7 & 7.17 \\
\hline$\xi$ & 342.5 & 4.69 & 340.8 & 5.54 & 330.2 & 10.41 \\
\hline \multicolumn{7}{|l|}{ Log-normal } \\
\hline$\mu$ & 5.50 & 0.058 & 5.50 & 0.060 & 5.56 & 0.080 \\
\hline$\sigma$ & 0.541 & 0.034 & 0.537 & 0.036 & 0.448 & 0.044 \\
\hline$\xi$ & 308.3 & 11.16 & 307.5 & 11.72 & 285.1 & 19.04 \\
\hline \multicolumn{7}{|l|}{ Wald } \\
\hline$\kappa$ & 298.7 & 13.4 & 299.7 & 13.9 & 304.5 & 19.1 \\
\hline$\lambda$ & 996.1 & 158.3 & $1,046.3$ & 179.8 & $1,603.3$ & 395.7 \\
\hline$\xi$ & 294.8 & 11.07 & 292.2 & 11.9 & 268.9 & 19.05 \\
\hline
\end{tabular}

Note-Censoring and truncation involved imposing a threshold at 1,000 msec. This resulted in discarding 19 RTs. $N=459$ correct RTs; see Figure 2, top.

ponents, computational problems will arise. Of course, if these shift parameters are constrained to be equal over the components, the mixture can be fit without such problems. For instance, Johnson et al. (1994) discussed a mixture of Weibulls, in which the shift parameter was constrained to be equal over the components. We did not pursue this possibility here.

We expected the error RTs to make up $25 \%$ of the data. In fact, we observed $233 /(233+669)=26 \%$. In the mixture distribution of RTs of the correct responses, we expected the mixing proportion to equal $25 / 75$, or $1 / 3$ [i.e., $25 \%$ of the correct response RTs were expected to be fast and $50 \%$ expected to be slow, so the mixing proportion was expected to equal $25 /(25+50)]$. We first estimated the parameters without equality constraints. The estimates are shown in Table 9. The mixing proportion was estimated at $.20(S E=.027)$. Next we introduced equality constraints. We specified that the ex-Gaussian parameters of the error distribution equal those of the fast component in the mixture. The LD $\chi^{2}$ test for these equalities equaled $7.6(d f=3, p=.06)$. We concluded that the equalities were tenable, but we note that the discrepancy observed in the parameter $\tau_{\mathrm{cf}}$ of the fast component was considerable: Without the constraints, we observed 11.1 $(S E=12.78)$, and with the constraints, we observed 45.1 $(S E=3.44)$. The mixing proportion estimate now equaled $.276(S E=.022)$. The standard error of .022 suggests that the mixing proportion deviated from the expected value of $1 / 3$. Finally, we carried out a LD test by retaining the equality constraints and fixing the mixing proportion to
$1 / 3$. The LD test equaled $\chi^{2}=6.0(d f=1 ; p=.014)$. We again concluded that the mixing proportion deviated from $1 / 3$, albeit slightly. Figure 3 displays the fitted distributions superimposed on the histograms.

Table 8

Illustration 2: Maximum Likelihood Estimates in Simultaneous Analyses of Error and Correct RTs, Subject to Right-Censoring of the Error RTs (Excluding RTs of 900 and $1,367 \mathrm{msec}$ )

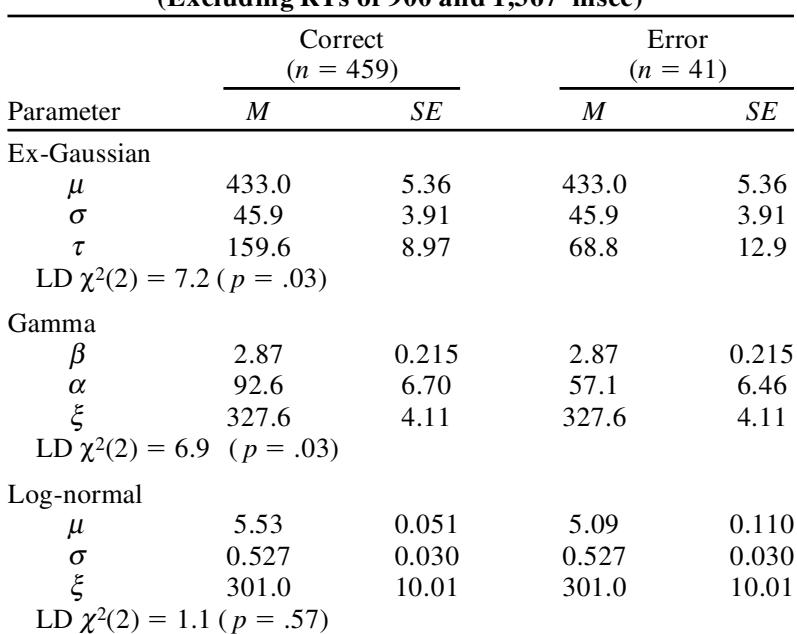

Note-Illustrative equality constraints were imposed on $\mu$ and $\sigma$ in the ex-Gaussian, $\beta$ and $\gamma$ in the gamma, and $\sigma$ and $\xi$ in the log-normal distributions. These constraints were tested by means of LD $\chi^{2}$ tests $(d f=2)$. 

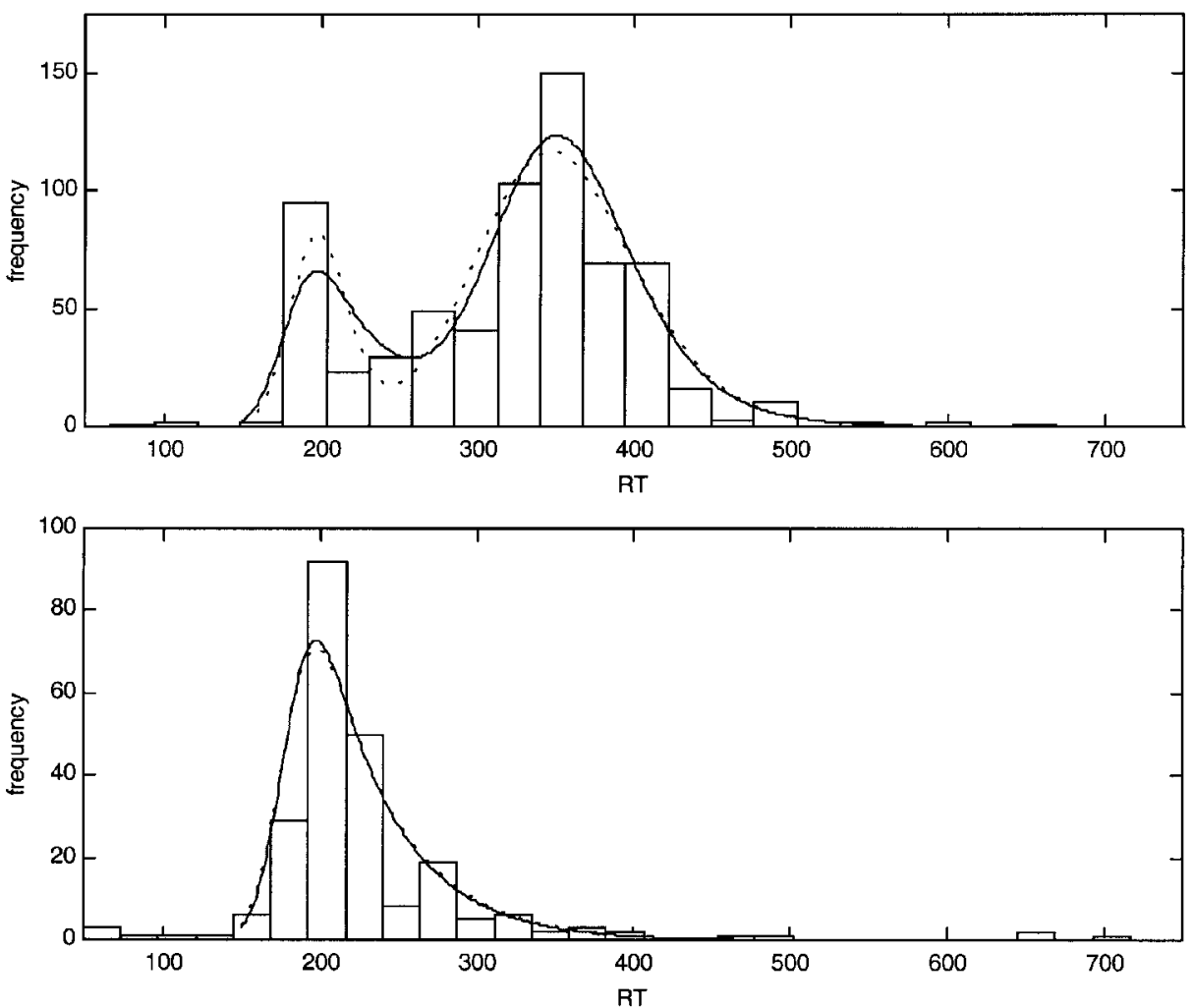

Figure 3. Illustration 3. Two-choice reaction time (RT) data subject to accuracy requirement (top, correct response RTs, $n=669$; bottom, error RTs, $n=233$ ). Superimposed are the fitted densities based on the parameter estimates in Table 9. Dashed lines represent the densities without equality constraints (see Table 9, Analysis 1); solid lines represent the densities with equality constraints (see Table 9, Analysis 2).

\section{DISCUSSION}

The aim of the present paper was to present a framework for distributionalRT analysis, based on ML estimation. The strength of this approach, as we see it, lies in the fact that once the required information (Table 1) of a given distribution has been programmed (see Appendix B), the parameters of this distribution may be estimated, given optional (left and/or right) truncation or censoring. In addition, any feasible finite mixture involving the distribution may be investigated. Multicase analyses and estimations subject to restrictions (bounds, equality constraints, or fixed parameters) may be carried out readily. We have limited our focus to ML estimation. Van Zandt (2000) demonstrated that this method of parameter estimation generally works well in distributional analyses. The results of our simulations support her conclusion.

The generality of the present strategy is also achieved by relying on the quasi-Newton routine NPSOL to maximize the loglikelihood function. Although we have had good experiences with NPSOL, other optimization methods may be used within the present framework (e.g., the Simplex algorithm; see Gill et al., 1981; Heathcote, 1996; Van Zandt, 2000). The IMSL MATH/library contains a variety of optimization routines. The quasi-Newton method is not necessarily the easiest way to obtain ML estimates in all situations. Also, an inconvenience is that this method requires admissible starting values that are not too far removed from the solution. This requirement implies that one has to be fairly well acquainted with one's data. We have relied on trial and error, graphical information (e.g., histograms), and, where readily available, moment estimators to obtain starting values. In addition, it is sometimes desirable to use several sets of starting values, to ensure that a given maximum is not local. Local maxima may be a serious problem in estimating parameters of mixture distributions (Everitt \& Hand, 1981).

Several workers in RT research have presented programs to carry out distributional analyses of RT data. Heathcote (1996) has presented a program that can estimate parameters of the ex-Gaussian, subject to optional censoring. Cousineau and Larochelle (1997) have presented a program that estimates parameters of the ex-Gaussian and the Weibull distributions. In addition, this program can be used for curve analysis - that is, analysis of changes in performance (on a given RT task) over time. Yantis et al. (1991) have presented a different approach to the analysis of finite mixtures of RT distributions, the multinomial ML 
Table 9

Illustration 3: Simultaneous Analysis of Correct $(N=669)$ and Incorrect $(N=233)$ RTs

\begin{tabular}{|c|c|c|c|c|}
\hline \multirow[b]{2}{*}{ Distribution } & \multicolumn{2}{|c|}{ Analysis $1^{*}$} & \multicolumn{2}{|c|}{ Analysis 2} \\
\hline & $M$ & $S E$ & $M$ & $S E$ \\
\hline \multicolumn{5}{|c|}{ Error RTs } \\
\hline \multicolumn{5}{|l|}{ Single ex-Gaussian } \\
\hline$\mu_{\text {err }}$ & 178.6 & 2.23 & 179.2 & 1.67 \\
\hline$\sigma_{\text {err }}$ & 15.6 & 1.53 & 15.0 & 1.15 \\
\hline$\tau_{\mathrm{err}}$ & 46.9 & 3.69 & 45.1 & 3.44 \\
\hline \multicolumn{5}{|c|}{ Correct RTs } \\
\hline \multicolumn{5}{|l|}{$\begin{array}{l}\text { Mixture of ex-Gaussians } \\
\text { Fast component }\end{array}$} \\
\hline$\mu_{\mathrm{cf}}$ & 189.1 & 2.23 & 179.2 & 1.67 \\
\hline$\sigma_{\mathrm{cf}}$ & 15.8 & 2.56 & 15.0 & 1.15 \\
\hline$\tau_{\mathrm{cf}}^{\mathrm{u}}$ & 11.1 & 12.78 & 45.1 & 3.44 \\
\hline Mixing proportion & .200 & .027 & .276 & .022 \\
\hline \multicolumn{5}{|l|}{ Slow component } \\
\hline$\mu_{\mathrm{cs}}$ & 322.3 & 4.47 & 327.4 & 3.74 \\
\hline$\sigma_{\mathrm{cs}}$ & 43.9 & 5.08 & 36.0 & 2.76 \\
\hline$\tau_{\mathrm{cs}}$ & 31.5 & 4.60 & 33.7 & 3.67 \\
\hline $\begin{array}{l}\log 1 \\
\operatorname{LD} \chi^{2}(3)=7.6(p=.06\end{array}$ & $-4,912.5$ & & $-4,916.3$ & \\
\hline
\end{tabular}

Note-Histograms are shown in Figure 3. Maximum likelihood parameter estimates of ex-Gaussian single component and ex-Gaussian mixture were estimated subject to censoring at 150 and $500 \mathrm{msec}$. Subscripts on parameters indicate errors (err), correct fast component (cf), and correct slow component (cs). *In Analysis 1, no equality constraints were imposed. In Analysis 2, the error RTs ex-Gaussian parameters were constrained to be equal to those of the fast component of the ex-Gaussian mixture. The equality constraints were judged to be tenable in view of the loglikelihood difference (LD) test.

mixture analysis. This method may be used when data are sampled both from the component distributions and from the mixture distributions. In the present approach, we compare distributions by estimating parameters of a given distribution in a multicase analysis with and without equality constraints (see Illustrations 2 and 3, Simulation 2). Yantis et al. created bins and cast the problem of comparing distributions in terms of a comparison of multinomial distributions and mixtures thereof. This approach has the great advantage that an explicit RT distribution need not be chosen and Pearson $\chi^{2}$ tests are valid when estimation and fitting are based on the categorized data (see Greenwood \& Nikulin, 1996). However, the number of bins is arbitrary and may affect the outcome of a given analysis (this applies equally to our use of the Pearson $\chi^{2}$ after full-information ML estimation). Also, this method is not well suited to investigating more subtle differences between distributions (e.g., distributional differences that are limited to shift, shape, or scale; see Illustration 2). Finally, as was noted by Yantis et al., the creation of bins necessarily involves a loss of information and, thus, of statistical power.

Ulrich and Miller (1994) presented an extensive study of censoring as a method of accommodating outliers. They demonstrated that the biasing effects of outliers are greatly reduced by censoring. As was noted above, they stressed that censoring is an approximate method, but one that does not present the computational difficulties of truncation. Ratcliff (1993) demonstrated that estimation may be carried out successfully, given truncation. However, Ratcliff (1993) limited his simulation study to sample sizes of 1,000 . Our experience agrees with Ulrich and Miller's (1994) findings that censoring is computationally less problematic than truncation. Ulrich and Miller (1994, p. 65) discussed the possibility of adopting a mixture approach to the accommodation of outliers. They stated that this approach requires one to specify a distribution for both fast and slow (outlying) RT distributions, as well as for the target distribution. This does not seem to be a feasible approach, especially since the outlying RTs, although influential (see Ulrich \& Miller, 1994), may actually account for a fraction of the total data. However, as was pointed out by an anonymous reviewer, the mixture approach to accommodating outliers may still be feasible under certain circumstances. Specifically, the reviewer mentioned the possibility of specifying a mixture of the target distribution (say, an ex-Gaussian) and a uniform distribution with fixed parameters. These parameters may be fixed to, say, the minimum and maximum observed RTs. This strategy, which may well be feasible, remains to be investigated in detail.

In conclusion, we believe that the present framework is worth considering, since it is efficient and flexible. We do emphasize that distributional analysis based on ML estimation is quite demanding. Anyone undertaking such analyses (1) has to be well acquainted with his or her data, (2) has to decide on a suitable distribution (see Yantis et al., 1991, for a method that does not require this), (3) has to provide, and sometimes vary, starting values, and (4) has to make sure that any obtained ML solution is acceptable in terms of goodness of fit (e.g., as assessed by the Pearson $\chi^{2}$ test), interpretability of the estimates, and magnitude of the standard error. Above, we have relied on the Pearson $\chi^{2}$ test to assess how well given distributions fit the data and on the LD test to compare nested models. Information criteria, such as the AIC (Akaike, 1974) and the BIC (Schwarz, 1978) may also be used in comparing (not necessarily nested) models. These criteria may be calculated readily, since they depend only on the loglikelihood, the number of estimated parameters, and in the case of BIC, the sample size. It is generally advisable to consider a variety of fit measures when comparing models.

\section{REFERENCES}

AKAIKe, H. (1974). A new look at statistical model identification. IEEE Transactions on Automatic Control, AU-19, 719-722.

Azzelini, A. (1996). Statistical inference based on the likelihood. London: Chapman and Hall.

Browne, M. W., \& CudEcK, R. (1993). Alternative ways of assessing model fit. In K. A. Bollen \& J. Scott Long (Eds.), Testing structural equation models. Newbury Park, CA: Sage.

BURBECK, S. L., \& LUCE, R. D. (1982). Evidence from auditory simple reaction times for both change and level detectors. Perception \& Psychophysics, 32, 117-133.

Colonius, H. (1995). The instance theory of automaticity: Why the Weibull? Psychological Bulletin, 102, 744-750.

Cousineau, D., \& Larochelle, S. (1997). PASTIS: A program for curve and distribution analysis. Behavior Research Methods, Instruments, \& Computers, 29, 542-548. 
DENNIS, J. E. \& SCHNABEL, R B. (1983). Numerical methods for unconstrained optimization and non-standard constraints. Englewood Cliffs, NJ: Prentice-Hall.

DOLAN, C. V. (2000). DISFIT: FORTRAN 77 program for distributional $R T$ analysis: Program documentation. Unpublished manuscript, University of Amsterdam, Department of Psychology.

Dolan, C. V., \& MolenaAR,P. C. M. (1991). A comparison of 4 methods of calculating standard errors in the analysis of covariance structure using normal theory ML estimation. British Journal of Mathematical \& Statistical Psychology, 47, 359-368.

Evans, M., Hastings, N., \& Peacock, B. (2000). Statistical distributions (3rd ed.). New York: Wiley.

EveritT, B. S., \& HANd, D. J. (1981). Finite mixture distributions. London: Chapman and Hall.

Gill, P. E., Murray, W., Saunders, M. A., \& Wright, M. H. (1986). User's guide for NPSOL (version 5.0-2). (Tech. rep. SOL 86-2). Stanford, CA: Stanford University, Department of Operations Research.

Gill, P. E., Murray, W., \& Wright, M. H. (1981). Practical optimization. London: Academic Press.

Greene, W. H. (1993). Econometric analysis (2nd ed.). New York: Macmillan.

GreEnwood,P. E., \& Nikulin, M. N. (1996). A guide to chi-square testing. New York: Wiley.

HEATHCOTE, A. (1996). RTSYS: A DOS application for the analysis of reaction time data. Behavior Research Methods, Instruments, \& Computers, 28, 427-445.

Heathcote, A., Popiel, S. J., \& Mewhort, D. J. K. (1991). Analysis of response time distributions: An example using the stroop task. Psychological Bulletin, 109, 340-347.

HEcK, A. (1997). Introduction to Maple (2nd ed.). New York: SpringerVerlag.

HocKLey, W. E. (1984). Analysis of response time distributions in the study of cognitive processes. Journal of Experimental Psychology: Learning, Memory, \& Cognition, 10, 598-615.

HoHLE, R. H. (1965). Inferred components of reaction times as functions of foreperiod duration. Journal of Experimental Psychology, 69, 382-386.

Johnson N. L., Kotz S., \& Balakrishnan, N. (1994). Continuousunivariate distributions (Vol. 1, 2nd ed.). New York: Wiley.

JöreSKOG, K. G. (1993). Testing structural equation models. In K. A. Bollen \& J. S. Long (Eds.), Testing structural equation models (pp. 294-316). Newbury Park, CA: Sage.

Kendall, M. G., \& Stuart, A. (1968). The advanced theory of statistics (Vol. 2, 3rd ed.). London: Griffin.

LogAN, G. D. (1992). Shapes of reaction-time distributions and shapes of learning curves: A test of the instance theory of automaticity. Journal of Experimental Psychology: Learning, Memory, \& Cognition, 18, 883-914.

Logan, G. D., \& Cowan, W. B. (1984). On the ability to inhibit thought and action: A theory of an act of control. Psychological Review, $\mathbf{9 1}$ 295-327.

LUCE, R. D. (1986). Response times: Their role in inferring elementary mental organization. New York: Oxford University Press.

Miller, I., \& Miller, M. (1999). John E. Freund's mathematical statistics (6th ed.). London: Prentice-Hall.

Moore, D. S. (1986). Tests of chi-squared type. In R. B. D'Agostino \& M. A. Stephens (Eds.), Goodness of fit techniques (pp. 63-95). New York: Dekker.

Neale, M. C., \& Miller, M. B. (1997). The use of likelihood-based confidence intervals in genetic models. Behavior Genetics, 27, 113-120.

RATCLIFF, R. (1979). Group reaction time distributions and an analysis of distribution statistics. Psychological Bulletin, 86, 190-214.

RatCLIFF, R. (1993). Methods for dealing with reaction time outliers. Psychological Bulletin, 114, 510-532.

Ratcliff, R., \& Murdock, B. B., JR. (1976). Retrieval processes in recognition memory. Psychological Review, 83, 190-214.
RAtcliff, R., VAN ZANDT, T., \& McKoon, G. (1999). Connectionis and diffusion models of reaction times. Psychological Review, 106, 261-300.

SChNipKe, D. L., \& Scrams, D. J. (1997). Modeling item response times with a two-state mixture model: A new method of measuring speededness. Journal of Educational Measurement, 34, 213-232.

SchwARZ, G. (1978). Estimating the dimension of a model. Annals of Statistics, 6, 461-464.

UlRICH, R., \& Miller, J. (1993). Information processing models generating lognormally distributed reaction times. Journal of Mathemat ical Psychology, 37, 513-525.

UlRICH, R, \& Miller, J. (1994). Effects of truncation of reaction time analysis. Journal of Experimental Psychology: General, 123, 34-80.

VAN ZANDT, T. (2000). How to fit a response time distribution. Psychonomic Bulletin \& Review, 7, 424-465.

Van Zandt, T., Colonius, H, \& Proctor, R. W. (2000). A comparison of two response time models applied to perceptual matching. Psychonomic Bulletin \& Review, 7, 208-256.

VAN ZANDT, T., \& RATCLIFF, R. (1995). Statistical mimicking of reaction time data: Single-process models, parameter variability, and mixtures. Psychonomic Bulletin \& Review, 2, 20-54.

Venzon, D. J., \& Moolgavkar, S. H. (1988). A method for computing profile-likelihood-based confidence intervals. Applied Statistics, 37, 87-94.

Visser, I., Raijmakers, M. E. J., \& Molenaar, P. C. M. (2000). Confidence intervals for hidden Markov model parameters. British Journal of Mathematical and Statistical Psychology, 53, 317-327.

Yantis S., Meyer, D. E., \& Smith, J. E. K. (1991). Analyses of multinomial mixture distributions: New tests for stochastic models of cognition and action. Psychological Bulletin, 110, 350-374.

\section{NOTES}

1. A program based on the present framework is available from www.psy.uva.nl/users/dolan. This is an executable, which runs in a DOS window under Windows. The Digital Visual FORTRAN 77 source code is available, but it is limited to parts written by the authors. The code includes the required information of the distributions mentioned above (see Table 1). The program calls the NPSOL subroutine (Gill et al., 1986) and various IMSL MATH/library and IMSL STAT/library subroutines. These are not included in the source. In addition to the six distribution functions mentioned, the program includes the Cauchy (Johnson et al., 1994) and, superfluously, since it is a special case of both the gamma and the Weibull distributions, the single-parameter exponential distribution function. The Cauchy is not a suitable RT distribution.

2 . The practice of first estimating parameters and subsequently carrying out the Pearson $\chi^{2}$ goodness-of-fit testing based on observed and expected bin counts is open to the following criticism. Under the null hypothesis, the calculated test statistic is often supposed to have a $\chi^{2}$ distribution with NBIN-NPAR- $1 d f \mathrm{~s}$ (NBIN is number of bins, NPAR is number of estimated parameters). However, was discussed by Greenwood and Nikulin (1996), the actual number of $d f \mathrm{~s}$ is unknown but is bounded by NBIN-NPAR-1 and NBIN-1. Greenwood and Nikulin presented a corrected $\chi^{2}$ goodness-of-fit test, which does not involve any loss of $d f$ for estimating parameters (i.e., $d f=\mathrm{NBIN}-1$ ). We have not incorporated this test in our program. Rather, we have followed Moore's advice that the known bounds on the $d f$ render the Pearson $\chi^{2}$ test useful in practice. Furthermore, when the number of bins is large and the number of estimated parameters is small, the Pearson $\chi^{2}$ test statistic approximates the $\chi^{2}$ null distribution quite closely (Moore, 1986, p. 68). Simulations based on the normal distribution and the single-parameter exponential show that the Pearson $\chi^{2}$ test does indeed work quite well when the number of bins is large, in the sense of good agreement between the expected Type I error rate and the observed Type I error rate. However the agreement was consistently better with Greenwood and Nikulin's test (Dolan, 2000). 


\section{APPENDIX A \\ Example of MAPLE-Generated FORTRAN Code for the Weibull Distribution Function, its Integral, and Derivatives With Respect to the Parameter $\alpha$}

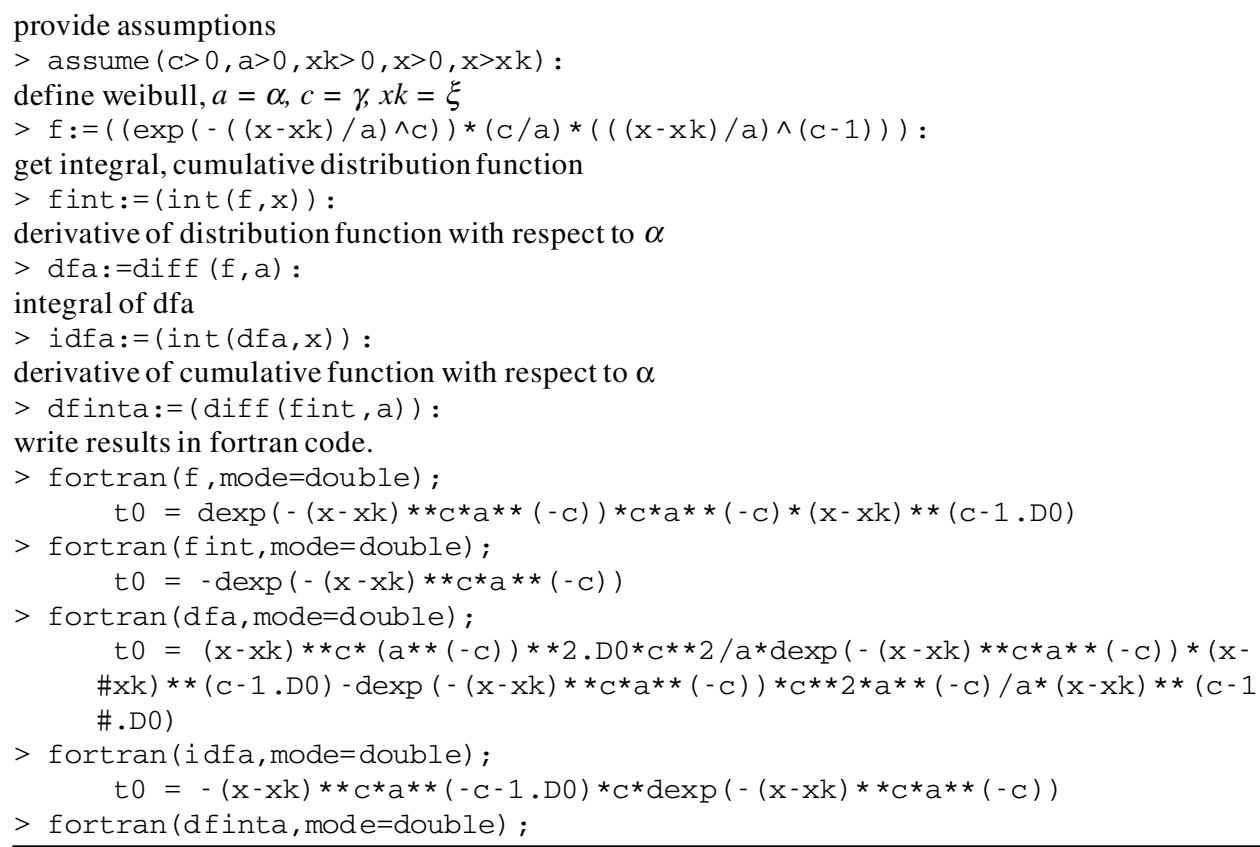

Note-As was expected, idfa and dfinta were equal. Although second-order derivatives were approximated in the present approach, they may be obtained readily [in MAPLE code, dfa2:= diff(f,a,a) and dfinta2:= diff(fint,a,a)].

\section{APPENDIX B}

Outline of Implementation of Present Framework in Pseudo Computer Code

For $j=1$ to n_of_cases

[first do calculations which do not depend on RT data

i.e., in case of truncation or censoring]

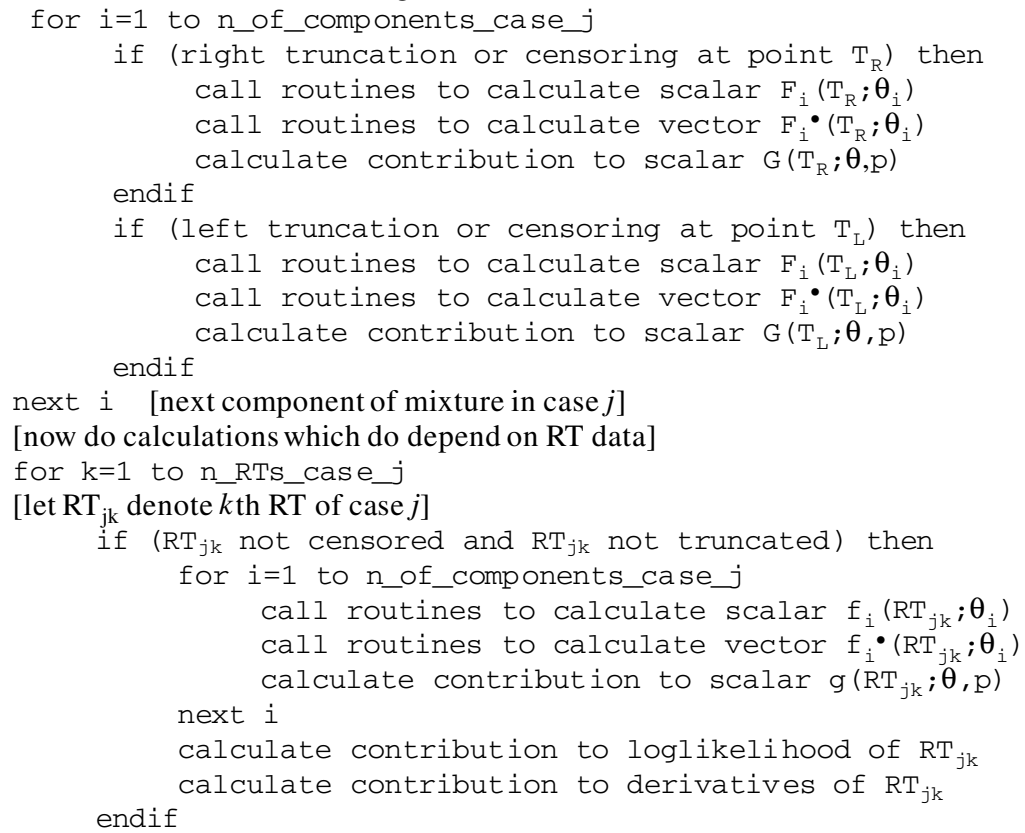


APPENDIX B (Continued)

next $\mathrm{k}$ [next RT of case $j]$

if (censoring in case $j$ ) then calculate contribution to loglikelihood given censoring calculate contribution to derivatives given censoring endif [see Tables 2C, 2E]

if (truncation in case $j$ ) then

calculate contribution to loglikelihood given truncation calculate contribution to derivative given truncation endif [see Tables 2B, 2D]

next $j$ [next case in analysis]

return

[to optimizer with log likelihood and vector of derivatives]

(Manuscript received September 27, 2000; revision accepted for publication January 18, 2002.) 